Copyright by the American Physical Society. Weng, Z. Y.; Ting, C. S.; Lee, T. K., "Mobile spin bags and their interaction in the spin-density-wave background," Phys. Rev. B 41, 1990 DOI: http://dx.doi.org/10.1103/PhysRevB.41.1990

\title{
Mobile spin bags and their interaction in the spin-density-wave background
}

\author{
Z. Y. Weng and C. S. Ting \\ Department of Physics and Texas Center for Superconductivity, University of Houston, Houston, Texas 77204-5506 \\ T. K. Lee \\ Department of Physics, Virginia Polytechnic Institute and State University, Blacksburg, Virginia 24061-4097
}

(Received 10 July 1989)

\begin{abstract}
The motion of one doped hole in the spin-density-wave background has been studied by the variational approach. A spin-bag solution for the doped hole has been obtained that applies in the weakand intermediate-coupling regime. The effective attraction between two spin bags with antiparallel spins has been shown to be $p$ - and $d$-wave like. The low-lying spin-flip excitations are found to be important both in the spin-bag effect of the doped holes and the effective attraction between them, which, in the latter case, are combined with the exchange of the simple-amplitude fluctuations to give a dominant contribution. On the other hand, the total attractive interaction is reduced by the Coulomb repulsion, which is restored in the presence of the "bag" effect.
\end{abstract}

\section{INTRODUCTION}

It is generally believed that the observed strong twodimensional (2D) antiferromagnetic spin correlations ${ }^{1}$ in the insulating phase of the oxide materials $\mathrm{La}_{2-x}(\mathrm{Sr}, \mathrm{Ba})_{x} \mathrm{CuO}_{4-y}$ and $\mathrm{YB}_{2} \mathrm{Cu}_{3} \mathrm{O}_{7-y}$ may provide an important clue to the understanding of high $T_{c}$ in the neighboring superconducting phase. Recently, the existence of strong spin correlations in the superconducting phase has also been reported. ${ }^{2}$ Naturally, it is tempting for one to get a hint of the high- $T_{c}$ superconducting state through doping, starting from the magnetic insulator parent. ${ }^{3-8}$ There are two ways to approach the $2 \mathrm{D}$ antiferromagnetic insulating state based on the single-band Hubbard model. One is from the strong-coupling, localized limit where one gets a Mott insulator in the halffilled case with one electron per $\mathrm{Cu}$ site, described by the antiferromagnetic Heisenberg Hamiltonian. The alternative way is from the itinerant approach, where the insulating spin-density-wave (SDW) state is present.

In the half-filled case, the mean-field SDW ground state will approach to the exact Néel state in the strongcoupling limit. In the same limit, the collective modes obtained in the itinerant picture ${ }^{8}$ agree with the spinwave excitations ${ }^{9}$ in the antiferromagnetic Heisenberg model. It is therefore reasonable to conjecture that the validity of the itinerant approach is not only restricted in the weak-coupling limit, it could be also applied to a moderately larger- $U$ regime where the realistic situation may be laid upon.

An essential point is the existence of the antiferromagnetic order, which presumably extends into the superconducting phase although it becomes short-range ordering. Based on this point of view, the spin-bag mechanism is proposed by Schrieffer, Wen, and Zhang (SWZ). ${ }^{4}$ When a hole is doped into the antiferromagnetic background, its motion should be renormalized to avoid disordering the background. But this causes an increase of the hole energy. From a more sophisticated point of view, the renormalization of the hole motion and its induced local distortion of background should be both present and reach a balance in energy. As a result one finds an entity of the quasihole surrounded by a local distortion of the SDW background moves around with charge $+e$ and spin index $\sigma= \pm \frac{1}{2}$, which is referred to as a "spin bag" by SWZ.

The spin-bag mechanism for superconductivity is intuitively based on the following continuum mean-field argument: Two spin bags attract each other to form the Cooper pair as the result that the two holes tend to share one common bag to lower their total energy. Quantitatively, the proper random-phase-approximation (RPA) approaches ${ }^{6-8}$ have shown that there is indeed a $d$-wavelike attraction between the quasiholes through exchanging the amplitude fluctuation. However, the amplitude fluctuation actually plays a less important role in the quasihole's self-energy, ${ }^{8}$ whereas the polaronic or spinbag effect is presumably important. Then one would naturally question the validity of such approaches ${ }^{6-8}$ to the effective potential, in which the effect of spin bag should also enter.

In the present paper, an alternative approach, i.e., the variational method, will be used and the results are justified by the Brillouin-Wigner perturbative approach. The Hubbard localized (on-site) interaction together with the existence of an SDW gap in the individual particlehole excitations make such an approach more efficient. An explicit wave function as well as the energy spectrum for spin bag are obtained in the intermediate coupling regime $U<W=8 t$. The spin-bag energy is in agreement with the numerical results of Su. ${ }^{5}$ The effective coupling between two spin bags is then discussed. Although the $p$-wave and $d$-wave-like attractive potential is still obtained, its behavior as a function of $U$ is quite different from the simple RPA approach presented in Refs. 6-8. We find that the low-energy spin-flip excitations, which 
are important to the spin-bag effect, also play an important role in the effective interaction through combining with the exchange of the simple amplitude fluctuations. The general conclusions in the present paper seem to be applicable at least qualitatively to the large doping region as long as the short-range antiferromagnetic order exists.

In Sec. II, the ground state of one doped hole in the SDW background is studied through the variational method which is justified by the Brillouin-Wigner perturbative approach. The wave function and energy spectrum for the doped hole are obtained. Then, the effective interaction between two spin bags is calculated in Sec. III, which is both qualitatively and quantitatively different from the conclusions obtained before. ${ }^{6-8}$ Finally, conclusions and discussions are presented in Sec. IV.

\section{A MOBILE SPIN BAG}

The two-dimensional Hubbard Hamiltonian with nearest-neighbor hopping on a square lattice is given by

$$
H=-t \sum_{\langle\iota, j\rangle_{\sigma}}\left(c_{i \sigma}^{\dagger} c_{j \sigma}+\text { H.c. }\right)+U \sum_{j} n_{j \uparrow} n_{j \downarrow} \text {. }
$$

In the (SDW) mean-field approximation, $H$ could be approximately diagonalized through the following canonical transformation: ${ }^{6}$

$$
\begin{aligned}
c_{j \sigma}=\sum_{\mathbf{k}}^{\prime} \frac{e^{i \mathbf{k} \cdot \mathbf{R}}}{\sqrt{N}}[ & \left(u_{\mathbf{k}}+\sigma p_{j} v_{\mathbf{k}}\right) \alpha_{\mathbf{k} \sigma} \\
& \left.+p_{j}\left(u_{\mathbf{k}}-\sigma p_{j} v_{\mathbf{k}}\right) \beta_{\mathbf{k} \sigma}\right] .
\end{aligned}
$$

And the exact Hamiltonian $H$ could be rewritten in two parts,

$$
H=H_{0}+H_{f},
$$

in which $H_{0}$ is the mean-field diagonalized part of $H$,

$$
\begin{aligned}
H_{0}=\mathrm{const}+\sum_{\mathbf{k}, \sigma}^{\prime}[ & \left(-E_{\mathbf{k}}-\mu\right) \alpha_{\mathbf{k} \sigma}^{\dagger} \alpha_{\mathbf{k} \sigma} \\
& \left.+\left(E_{\mathbf{k}}-\mu\right) \beta_{\mathbf{k} \sigma}^{\dagger} \beta_{\mathbf{k} \sigma}\right],
\end{aligned}
$$

where $\mu=-U / 2$ at the half-filled case. $H_{f}$ is neglected in the mean-field approximation. It includes the fluctuations above the mean-field ground state

$$
H_{f}=U \sum_{j} \delta n_{j \uparrow} \delta n_{j \downarrow},
$$

where $\delta n_{j \sigma}=n_{j \sigma}-\left\langle n_{j \sigma}\right\rangle_{0}$ and $\left\langle n_{j \sigma}\right\rangle_{0}$ is the average on the mean-field ground state.

In the canonical transformation (1.2), the $\mathbf{k}$-space operators $\alpha_{\mathbf{k} \sigma}$ and $\beta_{\mathrm{k} \sigma}$ describe, respectively, the lower and upper quasiparticle bands split by the SDW gap $\Delta$ and the wave vector $\mathbf{k}$ extends within the new Brillouin zone (i.e., the magnetic zone) which reduced to the half of the original one with the nesting vector $Q=( \pm \pi /$ $a, \pm \pi / a$ ) being a new reciprocal vector (see Fig. 1 of Ref. 6). The coefficients $u_{\mathrm{k}}$ and $v_{\mathrm{k}}$ in Eq. (1.2) are determined by

$$
u_{\mathrm{k}}=\left(\frac{1-\varepsilon_{\mathrm{k}} / E_{\mathrm{k}}}{2}\right)^{1 / 2},
$$

$$
v_{\mathrm{k}}=\left(\frac{1+\varepsilon_{\mathrm{k}} / E_{\mathrm{k}}}{2}\right)^{1 / 2},
$$

in which

$$
\begin{aligned}
& \varepsilon_{\mathbf{k}}=-2 t\left[\cos \left(k_{x} a\right)+\cos \left(k_{y} a\right)\right], \\
& E_{\mathbf{k}}=\left(\varepsilon_{\mathbf{k}}^{2}+\Delta^{2}\right)^{1 / 2},
\end{aligned}
$$

and the quantity $p_{j}$ appearing in Eq. (1.2) is defined by

$$
p_{j}=e^{i \mathbf{Q} \cdot \mathbf{R}},
$$

which has only two values: \pm 1 according to different lattice site $\mathbf{R}_{j}$. In fact, $p_{j}$ determines the two sublattices of the SDW state. This can be seen from the staggered magnetization moment $m_{j}$ at the lattice side $j$ whose expression in the mean-field theory is given by

$$
m_{j}=(2 \Delta / U) p_{j} .
$$

Thus one gets two sublattices with the total spin at each site either up or down, corresponding to $p_{j}=+1$ and $p_{j}=-1$, respectively.

Based on either sublattice, we could define a complete set of the Wannier-type states in the lattice space

$$
\begin{aligned}
& \alpha_{l \sigma}=\sum_{\mathbf{k}}^{\prime} \frac{e^{i \mathbf{k} \cdot \overline{\mathbf{R}}_{l}}}{\sqrt{N / 2}} \alpha_{\mathbf{k} \sigma}, \\
& \beta_{l \sigma}=\sum_{\mathbf{k}}^{\prime} \frac{e^{i \mathbf{k} \cdot \overline{\mathbf{R}}_{l}}}{\sqrt{N / 2}} \beta_{\mathbf{k} \sigma},
\end{aligned}
$$

where $\overline{\mathbf{R}}_{l}$ is the lattice vector for the given sublattice. It is easy to show that $\alpha_{\mathbf{k} \sigma}$ and $\beta_{\mathbf{k} \sigma}$ could be inversely expressed by $\alpha_{l \sigma}$ and $\beta_{l \sigma}$, which means the two sublattices are equivalent in defining the lattice representation (1.11). For symmetry, in the following we shall choose the sublattice such that $m_{j}>0(<0)$ in discussing the doped hole with spin up (down). The difference between the sublattices should be remembered only in the case involving two more doped holes with different spins.

According to the lattice representations (1.11a) and (1.11b), $H_{0}$ in Eq. (1.4) could be rewritten into the following form:

$$
\begin{aligned}
H_{0}= & \text { const }-\sum_{l m \sigma}^{\prime} t_{l m}\left(\alpha_{l \sigma}^{\dagger} \alpha_{m \sigma}-\beta_{l \sigma}^{\dagger} \beta_{m \sigma}\right) \\
& +\frac{U}{2} \sum_{l \sigma}^{\prime}\left(\alpha_{l \sigma}^{\dagger} \alpha_{l \sigma}+\beta_{l \sigma}^{\dagger} \beta_{l \sigma}\right)
\end{aligned}
$$

where the summations are over the given sublattice and the hopping integral $t_{l m}$ is defined by

$$
t_{l m}=\frac{2}{N} \sum_{\mathbf{k}}^{\prime} E_{\mathbf{k}} e^{i \mathbf{k} \cdot\left(\overline{\mathbf{R}}_{m}-\overline{\mathbf{R}}_{l}\right)} .
$$

The bare electron operator $c_{j \sigma}$ is expressed in the lattice representation according to the canonical transformations (1.2), (1.11a), and (1.11b) as follows:

$$
c_{j \sigma}=\sum_{l}^{\prime}\left[B_{\sigma j}(l) \alpha_{l \sigma}+p_{j} B_{-\sigma j}(l) \beta_{l \sigma}\right],
$$

in which $B_{\sigma_{J}}(l)$ is given by 


$$
B_{\sigma j}(l)=\frac{\sqrt{2}}{N} \sum_{\mathbf{k}}^{\prime} e^{i \mathbf{k} \cdot\left(\mathbf{R}_{j}-\overline{\mathbf{R}}_{l}\right)}\left(u_{\mathbf{k}}+\sigma p_{j} v_{\mathbf{k}}\right) .
$$

The mean-field ground state $|0\rangle$ in the half-filled case is an itinerant antiferromagnetic insulating state, with the lower quasiparticle band filled with electrons while the upper band is totally empty. When a hole is doped into such an SDW background, it is described by the quasihole state $|\mathbf{k} \sigma\rangle=\alpha_{\mathbf{k} \sigma}|0\rangle$ with the energy spectrum $E_{\mathrm{k}}-U / 2$ in the mean-field theory, which is an eigenstate of $H_{0}$. But beyond the mean-field approximation, one finds a coupling of the quasihole with the SDW background through $H_{f}$.

The motion of the doped hole is described by the Heisenberg equation

$$
\begin{aligned}
-i \frac{d}{d t} \alpha_{\mathbf{k} \sigma}(t)= & \left(E_{\mathbf{k}}-\frac{U}{2}\right) \alpha_{\mathbf{k} \sigma}(t) \\
& +\left[H_{f}(t), \alpha_{\mathbf{k} \sigma}(t)\right],
\end{aligned}
$$

where $\alpha_{\mathbf{k} \sigma}(t)$ and $H_{f}(t)$ are both in the Heisenberg representation. For one doped hole case, Eq. (1.16) will act on the half-filled SDW ground state. But we shall pursue SWZ's basic point of view that the spin-bag effect comes from the coupling of the doped hole with the mean-field SDW background. Thus in the following approach the half-filled true ground state will be replaced by the mean-field state $|0\rangle$. The correction to $|0\rangle$ due to the zero-point fluctuations will be neglected. Under this approximation, we study the renormalization effect on the quasihole due to its coupling with the excitations above the mean-field SDW ground state.

Hence, the doped hole's time-dependent state is determined by $\alpha_{\mathrm{k} \sigma}(t)|0\rangle$. In the absence of the commutator [ $\left.H_{f}(t), \alpha_{\mathrm{k} \sigma}(t)\right]$, Eq. (1.16) simply gives a phase factor to the time dependence of the state $\alpha_{\mathrm{k} \sigma}(t)|0\rangle$. The commutator $\left[H_{f}(t), \alpha_{\mathrm{k} \sigma}(t)\right]$ in Eq. (1.16) could be regarded as instant excitations at time $t$, induced by the doped hole in the SDW background. It is easy to verify

$\left[H_{f}(t), \alpha_{\mathbf{k} \sigma}(t)\right]|0\rangle=\sum_{l}^{\prime} \frac{e^{-i \mathbf{k} \cdot \overline{\mathbf{R}}_{l}}}{\sqrt{N / 2}} \hat{\phi}_{l \sigma}(t)|0\rangle$,

where

$$
\hat{\phi}_{l \sigma}=U \sum_{l^{\prime} m n}^{\prime} \sum_{j} p_{j} B_{\sigma J}(l) B_{\sigma j}(m) B_{\sigma J}(n) B_{-\sigma j}\left(l^{\prime}\right) \beta_{m-\sigma}^{\dagger} \alpha_{n \sigma} \alpha_{l^{\prime}-\sigma}
$$

As it has been noted before, the sublattice $\overline{\mathbf{R}}_{l}$ is chosen such that the on-site magnetization moment $m_{j=l}$ has the same sign as the doped hole's spin $\sigma$, i.e., $\sigma p_{j=1}=+1$ just for the reason of symmetry.

A basic excitation state is therefore obtained according to Eq. (1.17):

$\left|\phi_{\mathbf{k} \sigma}\right\rangle=\sum_{l}^{\prime} \frac{e^{-i \mathbf{k} \cdot \mathbf{R}_{J}}}{\sqrt{N / 2}} \hat{\phi}_{l \sigma}|0\rangle /\left(\left\langle 0\left|\hat{\phi}_{l \sigma}^{\dagger} \hat{\phi}_{l \sigma}\right| 0\right\rangle\right)^{1 / 2}$,

in which a particle-hole pair is excited around the doped hole as Eq. (1.18) shows. Actually, $\left|\phi_{\mathrm{k} \sigma}\right\rangle$ is the most important excitation state in the weak- and intermediatecoupling regime $(U<W=8 t)$. This point will be made more clearly later.

Then a variational state for a doped hole could be constructed by $|\mathbf{k} \sigma\rangle$ and $\left|\phi_{\mathbf{k} \sigma}\right\rangle$ as follows:

$$
\left|\psi_{\mathbf{k} \sigma}\right\rangle=\sin \theta_{\mathbf{k}}|\mathbf{k} \sigma\rangle-\cos \theta_{\mathbf{k}}\left|\phi_{\mathbf{k} \sigma}\right\rangle .
$$

By minimizing $\left\langle\psi_{\mathbf{k} \sigma}|H| \psi_{\mathbf{k} \sigma}\right\rangle, \theta_{\mathbf{k}}$ is determined by

$$
\tan 2 \theta_{\mathbf{k}}=-\frac{\left\langle\mathbf{k} \sigma\left|H_{f}\right| \phi_{\mathbf{k} \sigma}\right\rangle+\text { c.c. }}{\left\langle\phi_{\mathbf{k} \sigma}|H| \phi_{\mathbf{k} \sigma}\right\rangle-E_{0}-E_{\mathbf{k}}+U / 2},
$$

where $E_{0}=\langle 0|H| 0\rangle$. The quasihole's spectrum is shifted from $E_{\mathbf{k}}-U / 2$ to $\widetilde{E}_{\mathbf{k}}$ as follows:

$$
\widetilde{E}_{\mathbf{k}}=E_{\mathrm{k}}-\frac{U}{2}-\frac{\left\langle\mathbf{k} \sigma\left|H_{f}\right| \phi_{\mathbf{k} \sigma}\right\rangle+\text { c.c. }}{2} \cot \theta_{\mathrm{k}} .
$$

The function $B_{ \pm \sigma j}(l)$ appearing in Eq. (1.18) is a strongly localized function of $\mathbf{R}_{j}-\overline{\mathbf{R}}_{l}$ [Eq. (1.15)], which enables one to make the following expansion in $\phi_{l \sigma}[\mathrm{Eq}$. (1.18)]:

$$
\begin{aligned}
\hat{\phi}_{l \sigma}= & \beta_{l-\sigma}^{\dagger} \alpha_{l \sigma}\left(U \sum_{l^{\prime}}^{\prime} \sigma K_{0}\left(l, l^{\prime}\right) \alpha_{l^{\prime}-\sigma}\right) \\
& +U \sum_{l^{\prime}(\neq l)}^{\prime} \sigma K_{1}\left(l, l^{\prime}\right)\left(\beta_{l^{\prime}-\sigma}^{\dagger} \alpha_{l \sigma} \alpha_{l-\sigma}\right. \\
& \left.+\beta_{l-\sigma}^{\dagger} \alpha_{l^{\prime} \sigma} \alpha_{l-\sigma}+\beta_{l^{\prime}-\sigma}^{\dagger} \alpha_{l^{\prime} \sigma} \alpha_{l^{\prime}-\sigma}\right) \\
& +U \sum_{l^{\prime}(\neq l)}^{\prime} \sigma K_{2}\left(l, l^{\prime}\right) \beta_{l^{\prime}-\sigma}^{\dagger} \alpha_{l^{\prime} \sigma} \alpha_{l-\sigma}+\cdots,
\end{aligned}
$$

where

$$
\begin{aligned}
& K_{0}\left(l, l^{\prime}\right)=\sum_{j} \sigma p_{j} B_{\sigma j}^{3}(l) B_{-\sigma j}\left(l^{\prime}\right), \\
& K_{1}\left(l, l^{\prime}\right)=\sum_{j} \sigma p_{j} B_{\sigma j}^{2}(l) B_{\sigma j}\left(l^{\prime}\right) B_{-\sigma j}(l), \\
& K_{2}\left(l, l^{\prime}\right)=\sum_{j} \sigma p_{j} B_{\sigma j}(l) B_{\sigma j}^{2}\left(l^{\prime}\right) B_{-\sigma j}(l) .
\end{aligned}
$$

We denote $K_{00}=K_{0}(l, l) ; \quad$ and $K_{01}=K_{0}\left(l, l^{\prime}\right), \quad K_{11}$ $=K_{1}\left(l, l^{\prime}\right), K_{21}=K_{2}\left(l, l^{\prime}\right)$ for $l^{\prime}=$ nearest neighbor of $l$ (NN) in the sublattice; and so on. In Table I, the numerical values of the coefficients $K_{i j}$ have been shown for the

TABLE I. Numerical values of the coefficients $K_{i j}$ for the first four terms.

\begin{tabular}{rcclc}
\hline \hline$U$ & $K_{00}$ & $K_{01}$ & \multicolumn{1}{c}{$K_{11}$} & \multicolumn{1}{c}{$K_{21}$} \\
\hline $2 t$ & 0.276 & 0.029 & -0.021 & -0.012 \\
$5 t$ & 0.236 & 0.058 & -0.009 & -0.005 \\
$10 t$ & 0.148 & 0.045 & -0.002 & -0.001 \\
$20 t$ & 0.079 & 0.026 & $-2.5 \times 10^{-4}$ & $-1.5 \times 10^{-4}$ \\
\hline \hline
\end{tabular}


first several terms. We find that $K_{1}\left(l, l^{\prime}\right), K_{2}\left(l, l^{\prime}\right)$, and all of those which are not explicitly present in the expansion (1.23) are much less than $K_{00}$ in the whole region of $U$, and therefore, as a good approximation, one could retain the first term in the right-hand side of Eq. (1.23), i.e.,

$$
\hat{\phi}_{l \sigma} \simeq\left(U \sum_{l^{\prime}}^{\prime} \sigma K_{0}\left(l, l^{\prime}\right) \alpha_{l^{\prime}-\sigma}\right) \beta_{l-\sigma}^{\dagger} \alpha_{l \sigma} .
$$

Defining

$$
\begin{aligned}
& \Gamma_{0}=\left\langle\mathbf{k} \sigma\left|H_{f}\right| \phi_{\mathbf{k} \sigma}\right\rangle / U, \\
& \Gamma_{1}=\left\langle\phi_{\mathbf{k} \sigma}\left|H_{0}\right| \phi_{\mathbf{k} \sigma}\right\rangle-E_{0}+U / 2,
\end{aligned}
$$

and

$$
\Gamma_{2}=\left\langle\phi_{\mathrm{k} \sigma}\left|H_{f}\right| \phi_{\mathrm{k} \sigma}\right\rangle,
$$

then a direct calculation shows

$$
\begin{aligned}
& \Gamma_{0}=\left(\sum_{l}^{\prime} K_{0}^{2}(0, l)\right)^{1 / 2} ; \\
& \Gamma_{1}=3 t_{0}+\frac{1}{\Gamma_{0}^{2}} \sum_{l l^{\prime}\left(l \neq l^{\prime}\right)}^{\prime \prime} t_{l l^{\prime}} K_{0}(0, l) K_{0}\left(0, l^{\prime}\right) ; \\
& \Gamma_{2}=-U \sum_{j} B_{\sigma j}^{4}(0)+\frac{U}{\Gamma_{0}^{2}} \sum_{j} B_{\sigma j}^{2}(0) \\
& \times\left(\sum_{l}^{\prime} B_{-\sigma j}(l) K_{0}(0, l)\right)^{2},
\end{aligned}
$$

where $t_{0} \equiv t_{l l}$.

Therefore, according to Eqs. (1.19) - (1.25),

$$
\left|\psi_{\mathrm{k} \sigma}\right\rangle=\hat{\psi}_{\mathrm{k} \sigma}|0\rangle \text {, }
$$

where the spin-bag operator $\hat{\psi}_{\mathbf{k} \sigma}$ is defined by

$$
\hat{\psi}_{\mathbf{k} \sigma}=\sum_{l}^{\prime} \frac{e^{-l \mathbf{k} \cdot \overline{\mathbf{R}}_{l}}}{\sqrt{N / 2}}\left(\sin \theta_{\mathbf{k}}+\sigma \cos \theta_{\mathbf{k}} \beta_{l-\sigma}^{\dagger} \gamma_{l-\sigma}\right) \alpha_{l \sigma},
$$

in which

$$
\begin{aligned}
& \gamma_{l-\sigma}=\frac{1}{\Gamma_{0}} \sum_{l^{\prime}}^{\prime} K_{0}\left(l, l^{\prime}\right) \alpha_{l^{\prime}-\sigma}, \\
& \tan 2 \theta_{\mathbf{k}}=-\frac{2 \Gamma_{0} U}{\Gamma_{1}+\Gamma_{2}-E_{\mathbf{k}}},
\end{aligned}
$$

$\theta_{\mathbf{k}}$ is chosen such that $\tan \theta_{\mathbf{k}}>0$ and the spin bag's spectrum is given by

$$
\widetilde{E}_{\mathrm{k}}=E_{\mathrm{k}}-\frac{U}{2}-U \Gamma_{0} \cot \theta_{\mathrm{k}} .
$$

As Eqs. (1.32) and (1.33) show, $\widetilde{E}_{\mathrm{k}}$ is still a simple function of $E_{\mathrm{k}}$, with its lowest energy reached along the magnetic zone boundary (corresponding to $E_{\mathrm{k}}=\Delta$ ). The lowest energy of the spin-bag band as a function of $U / t$ is shown in Fig. 1 by a solid curve, in contrast with $\Delta-U / 2$, the quasihole's lowest energy which is represented by a dotted curve. The crossed curve in Fig. 1 represents a localized spin-bag solution which has been obtained in several numerical works ${ }^{5}$ within the inhomogeneous mean-field approximation. But the spin bag in the present paper is a mobile one which gains more kinetic energy and thus has lower energy as compared to the localized spin-bag solution, as Fig. 1 shows. On the other hand, Eq. (1.33) shows that the "spin-bag" effect not only greatly reduces the quasihole's energy, but also narrows the bandwidth. For example, the reduction of the bandwidth reaches to $50 \%$ at $U=5 t$.

To have a picture of the size of the spin bag, we note that the wave functions (1.30) are composed by a set of Wannier-type functions. The distribution of such a Wannier-type function centered at some site mainly spreads over to its four nearest-neighbor sites, consistent with the localized solution in the numerical approach. ${ }^{5}$ The small size of the spin bag comes from the quick convergence in the expansion of Eq. (1.23), where an approximate truncation has been made.

In obtaining the spin-bag solution [Eqs. (1.29)-(1.33)], only one important excitation $\left|\phi_{\mathrm{k} \sigma}\right\rangle$ is involved in the variational state (1.20). To justify our solution, we introduce the following Brillouin-Wigner expansion: ${ }^{10}$

$$
\widetilde{E}_{\mathbf{k}}=E_{\mathbf{k}}-\frac{U}{2}+\left\langle\mathbf{k} \sigma|| H_{f}+H_{f} \frac{1}{E_{0}+\widetilde{E}_{\mathbf{k}}-H_{0}} H_{f}+H_{f} \frac{1}{E_{0}+\widetilde{E}_{\mathbf{k}}-H_{0}} H_{f} \frac{1}{E_{0}+\widetilde{E}_{\mathbf{k}}-H_{0}} H_{f}+\left.\cdots\right|^{\prime} \mid \mathbf{k} \sigma\right\rangle .
$$

The prime on the large parentheses in Eq. (1.34) means that $|\mathbf{k} \sigma\rangle$ should not appear as an intermediate state in the expansion.

If only $\left|\phi_{\mathbf{k} \sigma}\right\rangle$ is retained in all the intermediate states of the Brillouin-Wigner expansion (1.34), then one again gets the results of Eq. (1.33) after using

$$
\left\langle\phi_{\mathbf{k} \sigma}\left|\frac{1}{E_{0}+\widetilde{E}_{\mathbf{k}}-H_{0}}\right| \phi_{\mathbf{k} \sigma}\right\rangle \simeq \frac{1}{E_{0}+\widetilde{E}_{\mathbf{k}}-\left\langle\phi_{\mathbf{k} \sigma}\left|H_{0}\right| \phi_{\mathbf{k} \sigma}\right\rangle} .
$$

The approximation (1.35) is equivalent to say that the effect of the hopping terms in $H_{0}$ [Eq. (1.12)] is not so im- portant. With a large $U$, this is certainly the case as

$$
t_{l l^{\prime}}\left(l \neq l^{\prime}\right) / t_{0} \sim 1 / U^{3} .
$$

At $U=2 t$, the error brought by the relation (1.35) to $\widetilde{E}_{\mathrm{k}}$ is $\sim 15 \%$ as estimated by the perturbative method. Thus as long as $U$ is not sufficiently small, the approximation (1.35) is reasonable. The propagator

$$
1 /\left(E_{0}+\widetilde{E}_{\mathrm{k}}-H_{0}\right)
$$

acting on $\left|\phi_{\mathbf{k} \sigma}\right\rangle$ will also produce other intermediate states and make the spin-bag size spread out, due to the hopping processes. But we find such an effect is similarly 


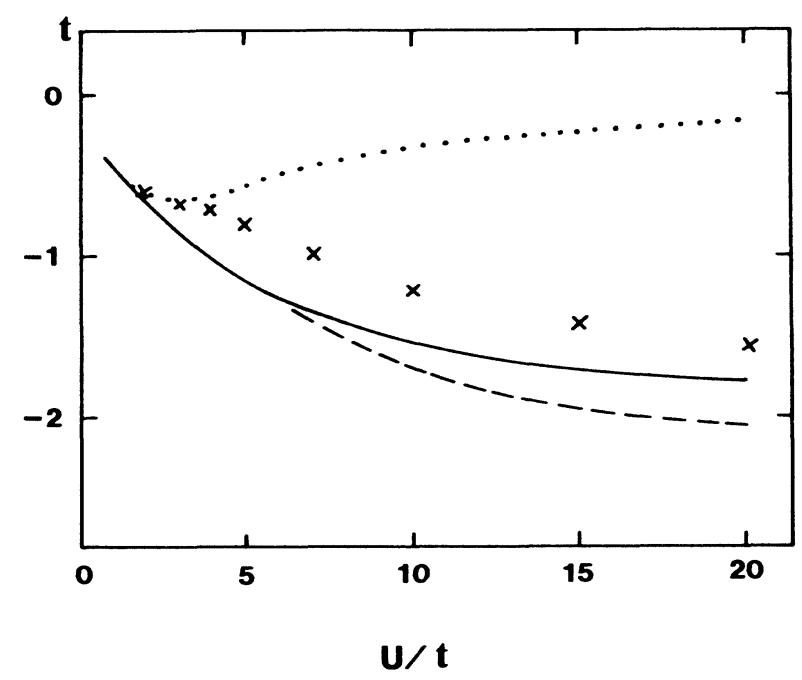

FIG. 1. The lowest energy of the spin-bag band vs $U$ is presented by the solid curve, in contrast with the dotted curve, $\Delta-U / 2$, the mean-field quasihole's energy along the magnetic zone boundary. The crossed curve is the localized spin-bag energy obtained by the numerical approach (Ref. 5). The dashed curve gives a higher order correction to the spin-bag solution Eq. (1.33) [see the context below Eq. (1.36)].

negligible provided $U$ is not too small.

Under the above-described approximations to obtain the spin-bag energy (1.33), all other matrix elements are neglected in the Brillouin-Wigner expansion (1.34) except $\left\langle\mathbf{k} \sigma\left|H_{f}\right| \phi_{\mathbf{k} \sigma}\right\rangle$ and $\left\langle\phi_{\mathbf{k} \sigma}\left|H_{f}\right| \phi_{\mathbf{k} \sigma}\right\rangle$. The contribution of the matrix element $\left\langle\phi_{\mathbf{k} \sigma}\left|\boldsymbol{H}_{f}\right| \phi_{\mathbf{k} \sigma}\right\rangle$ to $\widetilde{E}_{\mathbf{k}}$ is quite important. We have noted that $\left\langle\phi_{\mathbf{k} \sigma}\right\rangle$ describes a doped hole tightly accompanied by a particle-hole pair excited from the SDW background, according to Eqs. (1.19) and (1.25). In the process related to $\left\langle\phi_{\mathbf{k} \sigma}\left|H_{f}\right| \phi_{\mathbf{k} \sigma}\right\rangle$, one finds a strong coupling between the hole and the particle which carry opposite spins. This means that the contribution coming from the spin-flip components $\left(\sum \beta_{-\sigma}^{\dagger} \alpha_{\sigma}\right)$ is dominant in the self-energy of the spin bag. The physical picture is simple: A particle-hole pair is excited by the doped hole which actually is a charge excitation (excitation energy $>2 \Delta$ ). To decrease its energy cost, the excited particle has to couple with the doped hole strongly and closely accompanies it, forming an "exciton" which is related to the low-lying spin-flip excitation.

$\left|\phi_{\mathbf{k} \sigma}\right\rangle$ is deduced from $\dot{\alpha}_{\mathbf{k} \sigma}|0\rangle$ or $\left[H_{f}, \alpha_{\mathbf{k} \sigma}\right]|0\rangle$. More excitation states could be obtained from $\ddot{\alpha}_{\mathbf{k} \sigma}|0\rangle, \dddot{\alpha}_{\mathbf{k} \sigma}|0\rangle$, etc., in which terms like $\left[H_{f},\left[H_{f}, \alpha_{\mathbf{k} \sigma}\right]\right]|0\rangle$, and so on, are involved. According to the localized property of $B_{ \pm \sigma j}(l)$, one could find a straightforward way that except for $\left\langle\phi_{\mathbf{k} \sigma}\right\rangle$, the only important excited state coming from $\left[H_{f},\left[H_{f}, \alpha_{\mathbf{k} \sigma}\right]\right]|0\rangle$ is given by

$$
|\widetilde{\phi}\rangle \propto \sum_{l}^{\prime} \frac{e^{-i \mathbf{k} \cdot \overline{\mathbf{R}}_{l}}}{\sqrt{N / 2}} \hat{\chi}_{l \sigma}|0\rangle
$$

where

$$
\hat{\chi}_{l \sigma}=\sum_{l^{\prime}}^{\prime} \sigma K_{0}\left(l, l^{\prime}\right) \sum_{j} \sum_{m, n, p}^{\prime} p_{j} B_{\sigma j}(p) B_{-\sigma j}(m) B_{-\sigma j}(n) B_{-\sigma j}\left(l^{\prime}\right) \beta_{l-\sigma}^{\dagger} \alpha_{l \sigma} \beta_{m \sigma}^{\dagger} \alpha_{n-\sigma} \alpha_{p \sigma}|0\rangle .
$$

$|\hat{\phi}\rangle$ involves two particle-hole pairs excited from the background by the doped hole. Including both $\left|\phi_{\mathbf{k} \sigma}\right\rangle$ and $|\widetilde{\phi}\rangle$ in all the intermediate states in Eq. (1.34), we obtain a correction to Eq. (1.33). The dashed curve in Fig. 1 shows such a correction to the lowest energy of spin-bag spectrum. One finds that it is negligible in the regime $U\langle W=8 t$. As $\mid \widetilde{\phi}\rangle$ includes the spin-flip components $\left(\Sigma \beta_{\downarrow}^{\dagger} \alpha_{\uparrow}\right.$ and $\left.\Sigma \beta_{\uparrow}^{\dagger} \alpha_{\downarrow}\right)$ whose excitation energy decreases in the large $U$ regime, the correction coming from $|\widetilde{\phi}\rangle$ could become non-negligible at the strong-coupling regime $U \geq W$ as Fig. 1 shows. With the increase of $U$, one can expect the intermediate states with many particlehole excitations will be involved which result in an enlarging spin disorder around the doped hole. ${ }^{11}$ Therefore, we obtained a fairly good spin-bag solution in the intermediate-coupling regime $U<W=8 t$, in agreement with the conjecture of SWZ. ${ }^{4}$

\section{THE EFFECTIVE INTERACTION}

In Sec. II, the spin-bag solution is obtained for a single hole doped in the SDW background. The spin-bag opera- tor $\hat{\psi}_{\mathbf{k} \sigma}$ [Eq. (1.30)] satisfies, generally, the following equation:

$$
\left[H, \hat{\psi}_{\mathbf{k} \sigma}\right]=\widetilde{E}_{\mathbf{k}} \hat{\psi}_{\mathbf{k} \sigma}+\widehat{A}_{\mathbf{k} \sigma} .
$$

What we have done in the last section is equivalent to neglecting $\hat{A}_{\mathbf{k} \sigma}$. Therefore Eq. (2.1) is approximately diagonalized in the single hole case. In the presence of two or more spin bags, however, $\hat{A}_{\mathbf{k} \sigma}$ will play a role in the interaction between these spin bags.

To consider the interaction between spin bags, the basic state for two free spin bags is defined in the subspace of total zero-momentum and antiparallel spin (the case for the parallel spin can be similarly considered):

$$
|\mathbf{k} \uparrow,-\mathbf{k} \downarrow\rangle=\widehat{\psi}_{\mathbf{k} \uparrow} \hat{\psi}_{-\mathbf{k} \downarrow}|0\rangle .
$$

The matrix element $\left\langle\mathbf{k}^{\prime} \uparrow,-\mathbf{k}^{\prime} \downarrow|H| \mathbf{k} \uparrow,-\mathbf{k} \downarrow\right\rangle$ can be expressed as

$$
\begin{aligned}
& \left\langle\mathbf{k}^{\prime} \uparrow,-\mathbf{k}^{\prime} \downarrow|H| \mathbf{k} \uparrow,-\mathbf{k} \downarrow\right\rangle \\
& \quad=\left(2 \widetilde{E}_{\mathbf{k}}+E_{0}\right) \cdot\left\langle\mathbf{k}^{\prime} \uparrow,-\mathbf{k}^{\prime} \downarrow \mid \mathbf{k} \uparrow,-\mathbf{k} \downarrow\right\rangle+H_{\mathbf{k}^{\prime}, \mathbf{k}}^{\prime},
\end{aligned}
$$

where the interaction matrix element $H_{\mathbf{k}^{\prime}, \mathbf{k}}^{\prime}$ is determined by 


$$
\begin{aligned}
H_{\mathbf{k}^{\prime}, \mathbf{k}}^{\prime}= & \left\langle\mathbf{k}^{\prime} \uparrow,-\mathbf{k}^{\prime} \downarrow\left|\hat{A}_{\mathbf{k} \uparrow} \hat{\psi}_{-\mathbf{k} \downarrow}\right| 0\right\rangle \\
& +\left\langle\mathbf{k}^{\prime} \uparrow,-\mathbf{k}^{\prime} \downarrow\left|\hat{\psi}_{\mathbf{k} \uparrow} \hat{A}_{-\mathbf{k} \downarrow}\right| 0\right\rangle \\
& +\left\langle\mathbf{k}^{\prime} \uparrow,-\mathbf{k}^{\prime} \downarrow\left|\hat{\psi}_{\mathbf{k} \uparrow} \hat{\psi}_{-\mathbf{k} \downarrow} H_{f}\right| 0\right\rangle .
\end{aligned}
$$

$\hat{A}_{\mathbf{k} \sigma}$ defined in Eq. (2.1) plays a central role in the first two terms on the right-hand side of Eq. (2.4), while the last term represents a correlation between two spin bags through absorbing a virtual background fluctuation above the mean-field state.

In the approach to a single spin bag, we have chosen different sublattices for different spins [e.g., for the case of spin up (down), the sublattice is chosen such that $\left.p_{j}=e^{i \mathrm{Q} \cdot \mathbf{R}_{j}}=+1(-1)\right]$. In the following, the sublattice with $p_{j}=+1$ will be denoted by the lattice vector $\overline{\mathbf{R}}_{l}$, while for the sublattice with $p_{j}=-1$, it is defined by the lattice vector $\widetilde{\mathbf{R}}_{l}$ and the corresponding Wannier-type quasiparticle operators are denoted by $\widetilde{\alpha}_{l \sigma} \widetilde{\beta}_{l \sigma}$, etc., and the function defined in Eq. (1.15) is rewritten as $\widetilde{B}_{\sigma l}(l)$ for distinguishing.
It should be noted that the two-free-spin-bag state $|\mathbf{k} \uparrow,-\mathbf{k} \downarrow\rangle$ is not exactly orthogonal for different $\mathbf{k}$ 's as $\hat{\psi}_{\mathbf{k} \sigma}$ is a composite operator. Actually, one could find an exchange process giving a nonzero contribution to $\left\langle\mathbf{k}^{\prime} \uparrow,-\mathbf{k}^{\prime} \downarrow \mid \mathbf{k} \uparrow,-\mathbf{k} \downarrow\right\rangle$ for $\mathbf{k} \neq \mathbf{k}^{\prime}$. In Appendix A, however, such a contribution is shown to be proportional to $\cos ^{4} \theta$ in the region close to the magnetic zone boundary with $\left|\varepsilon_{\mathbf{k}}\right|,\left|\varepsilon_{\mathbf{k}^{\prime}}\right|<\Delta$, where $\cos \theta$ is the coefficient appearing in the spin-bag operator $\hat{\psi}_{\mathbf{k} \sigma}$ [Eq. (1.30)]. $\cos \theta$ is equal to zero as $U \rightarrow 0$. In the moderate $U$ regime, the order of $\cos ^{4} \theta$ will be neglected. To be consistent with such an approximately orthogonal condition, $H_{\mathbf{k}^{\prime}, \mathbf{k}}^{\prime}$ is meaningful only to the order which is lower than $\widetilde{E}_{\mathbf{k}} \cos ^{4} \theta$ as Eq. (2.3) shows. Therefore, the contribution in $H_{\mathbf{k}^{\prime}, \mathbf{k}}^{\prime}$ of Eq. (2.4) will be arranged in the power of $\cos \theta$ and retained up to the order of $\cos ^{2} \theta$.

In the scheme presented in Appendix B, $H_{\mathbf{k}^{\prime}, \mathbf{k}}^{\prime}$ could be straightforwardly calculated according to Eqs. (2.4) and (2.1). With the condition of $\left|\varepsilon_{k}\right|,\left|\varepsilon_{k^{\prime}}\right|<\Delta$ and to the order of $\cos ^{2} \theta$, one obtains according to Eqs. (B2), (B9), and (B10) in Appendix B

$$
\begin{aligned}
& H_{\mathbf{k}^{\prime}, \mathbf{k}}^{\prime}=-\sin \theta_{\mathbf{k}} \cos \theta_{\mathbf{k}} \sin \theta_{\mathbf{k}^{\prime}} \cos \theta_{\mathbf{k}^{\prime}} \frac{4}{N^{2}} \sum_{l l^{\prime}}^{\prime} e^{-i\left(\mathbf{k}-\mathbf{k}^{\prime}\right) \cdot\left(\overline{\mathbf{R}}_{l}-\overline{\mathbf{R}}_{l^{\prime}}\right)} \\
& \times U\left\{2 \sum_{j} B_{+j}(l) \widetilde{B}_{-j}\left(l^{\prime}\right) A_{j}(l) \widetilde{A}_{j}\left(l^{\prime}\right)-D_{l, l^{\prime}} \sum_{j} B_{+j}(l) \widetilde{B}_{-j}\left(l^{\prime}\right)\right. \\
& \times\left[A_{j}(l)\left(\frac{u_{\mathbf{k}^{\prime}}+p_{j} v_{\mathbf{k}^{\prime}}}{\sqrt{2}} e^{i \mathbf{k}^{\prime} \cdot\left(\mathbf{R}_{j}-\overline{\mathbf{R}}_{l}\right)}+\frac{u_{\mathbf{k}}+p_{j} v_{\mathbf{k}}}{\sqrt{2}} e^{-i \mathbf{k} \cdot\left(\mathbf{R}_{j}-\overline{\mathbf{R}}_{l}\right)}\right]\right. \\
& \left.\left.+\tilde{A}_{j}\left(l^{\prime}\right)\left[\frac{u_{\mathbf{k}^{\prime}}-p_{j} v_{\mathbf{k}^{\prime}}}{\sqrt{2}} e^{-i \mathbf{k}^{\prime} \cdot\left(\mathbf{R}_{j}-\tilde{\mathbf{R}}_{l^{\prime}}\right)}+\frac{u_{\mathbf{k}}-p_{j} v_{\mathbf{k}}}{\sqrt{2}} e^{i \mathbf{k} \cdot\left(\mathbf{R}_{j}-\tilde{\mathbf{R}}_{l^{\prime}}\right)}\right)\right]\right\} \\
& -\left(\sin ^{2} \theta_{\mathbf{k}} \cos ^{2} \theta_{\mathbf{k}^{\prime}}+\sin ^{2} \theta_{\mathbf{k}^{\prime}} \cos ^{2} \theta_{\mathbf{k}}\right) \frac{4}{N^{2}} \sum_{l, l^{\prime}}^{\prime} e^{-i\left(\mathbf{k}-\mathbf{k}^{\prime}\right) \cdot\left(\tilde{\mathbf{R}}_{l}-\tilde{\mathbf{R}}_{l^{\prime}}\right)} \cdot U \sum_{j} B_{+j}(l) \widetilde{B}-j\left(l^{\prime}\right) A_{j}(l) \widetilde{A}_{j}\left(l^{\prime}\right),
\end{aligned}
$$

in which $D_{l, l^{\prime}}, A_{j}(l)$, and $\widetilde{A}_{j}\left(l^{\prime}\right)$ are all strongly localized functions and defined by

$$
\begin{aligned}
& D_{l, l^{\prime}}=\frac{1}{\Gamma_{0}} \sum_{j} p_{j} B_{+j}^{3}(l) \widetilde{B}_{-j}\left(l^{\prime}\right), \\
& A_{j}(l)=\frac{1}{\Gamma_{0}} \sum_{j_{1}} p_{j_{1}} B_{+j_{1}}^{3}(l) \sum_{l^{\prime}}^{\prime} \widetilde{B}_{-j}\left(l^{\prime}\right) \widetilde{B}_{-j_{1}}\left(l^{\prime}\right) \\
& \widetilde{A}_{j}\left(l^{\prime}\right)=\frac{1}{\Gamma_{0}} \sum_{j_{1}}\left(-p_{j_{1}}\right) \widetilde{B}_{-j_{1}}^{3}\left(l^{\prime}\right) \sum_{l}^{\prime} B_{+j}(l) B_{+j_{1}}(l) .
\end{aligned}
$$

Such an effective potential is found to be the nearestneighbor interaction, that is, only $\widetilde{\mathbf{R}}_{l^{\prime}}=\widetilde{\mathbf{R}}_{l}+a( \pm \mathbf{i}, \pm \mathbf{j})$ is important in the summations over $l$ and $l^{\prime}$ in Eq. (2.5). On the other hand, along the magnetic zone boundary, $u_{k} \sim u_{k} \sim 1 / \sqrt{2}$, and one could approximately replace, for example,

$$
\left[\left(u_{\mathbf{k}}+p_{j} v_{\mathbf{k}}\right) / \sqrt{2}\right] e^{-i \mathbf{k} \cdot\left(\mathbf{R}_{j}-\tilde{\mathbf{R}}_{l}\right)}
$$

by the factor $\left(1+p_{j}\right) / 2$ in Eq. $(2.5)$ as only $\mathbf{R}_{j}=\overline{\mathbf{R}}_{l}$ has non-negligible contribution in the summation over $j$. Therefore, the effective interaction of Eq. (2.5) is reduced to the following form:

$$
H_{\mathbf{k}^{\prime}, \mathbf{k}}^{\prime}=-\frac{\cos \left(k_{x}^{\prime}-k_{x}\right) a+\cos \left(k_{y}^{\prime}-k_{y}\right) a}{2}\left(\frac{1}{N}\right) \boldsymbol{V},
$$

where

$$
\begin{aligned}
& V=8\left(\sin ^{2} \theta\right)\left(\cos ^{2} \theta\right) U \\
& \times \sum_{j}\left[4 B_{+j}(l) \widetilde{B}_{-j}\left(l^{\prime}\right) A_{j}(l) \widetilde{A}_{j}\left(l^{\prime}\right)-2 B_{+j}^{2}(l) \widetilde{B}_{-j}^{2}\left(l^{\prime}\right)\right. \\
& \left.\quad-2 D_{l, l^{\prime}}\left(\frac{1+p_{j}}{2} A_{j}(l)+\frac{1-p_{j}}{2} \widetilde{A}_{j}\left(l^{\prime}\right)\right]\right],
\end{aligned}
$$

in which $l^{\prime}$ is the nearest-neighbor site of $l$ in the original lattice ad $\theta_{\mathbf{k}} \simeq \theta_{\mathbf{k}}^{\prime}$ have been replaced approximately by $\theta \equiv \theta\left(\varepsilon_{\mathbf{k}}=0\right)$. 
By using the following $s$-wave-like, $p$-wave-like, and $d$ wave-like symmetry functions

$$
\begin{aligned}
& g_{s}(\mathbf{k})=\frac{1}{2}\left[\cos \left(k_{x} a\right)+\cos \left(k_{y} a\right)\right] ; \\
& g_{p}(\mathbf{k})=\frac{1}{2}\left[\sin \left(k_{x} a\right)+\sin \left(k_{y} a\right)\right] ; \\
& g_{p}^{\prime}(\mathbf{k})=\frac{1}{2}\left[\sin \left(k_{x} a\right)-\sin \left(k_{y} a\right)\right] ; \\
& g_{d}(\mathbf{k})=\frac{1}{2}\left[\cos \left(k_{x} a\right)-\cos \left(k_{y} a\right)\right],
\end{aligned}
$$

one has

$$
\begin{aligned}
& \frac{\cos \left(k_{x}^{\prime}-k_{x}\right) a+\cos \left(k_{y}^{\prime}-k_{y}\right) a}{2} \\
& =g_{s}(\mathbf{k}) g_{s}\left(\mathbf{k}^{\prime}\right)+g_{p}(\mathbf{k}) g_{p}\left(\mathbf{k}^{\prime}\right) \\
& +g_{p}^{\prime}(\mathbf{k}) g_{p}^{\prime}\left(\mathbf{k}^{\prime}\right)+g_{d}(\mathbf{k}) g_{d}\left(\mathbf{k}^{\prime}\right) .
\end{aligned}
$$

Around the magnetic zone boundary, it is easy to check $g_{s}(\mathbf{k}) g_{s}\left(\mathbf{k}^{\prime}\right) \simeq 0$ and thus $H_{\mathbf{k}^{\prime}, \mathbf{k}}^{\prime}$ has chiefly the $p$-wave and $d$-wave-like components in this region, which in fact is the direct consequence of the existence of two sublattices.

According to the interactive potential $V$ [Eq. (2.10)] and the spectrum $\widetilde{E}_{k}$ [Eq. (1.33)], the binding energy of two spin bags could be calculated in terms of Eq. (32) of Ref. 6. $H_{\mathbf{k}^{\prime}, \mathbf{k}}$ in Eq. (2.9) is derived in the region of $\left|\varepsilon_{\mathbf{k}}\right|,\left|\varepsilon_{\mathbf{k}^{\prime}}\right|<\Delta$. Around $\left|\varepsilon_{\mathbf{k}}\right| \simeq \Delta$, the change in $\widetilde{E}_{\mathbf{k}}$ is quite large as compared to the binding energy and it thus provides a natural cutoff-the states in the region $\left|\varepsilon_{\mathbf{k}}\right| \geq \Delta$ contribute little to the bound state of two spin bags due to a comparatively large enhancement of the "kinetic energy" $\widetilde{E}_{\mathrm{k}}$ in this region. Thus, although the effective potential is the nearest-neighbor interaction, the size of the

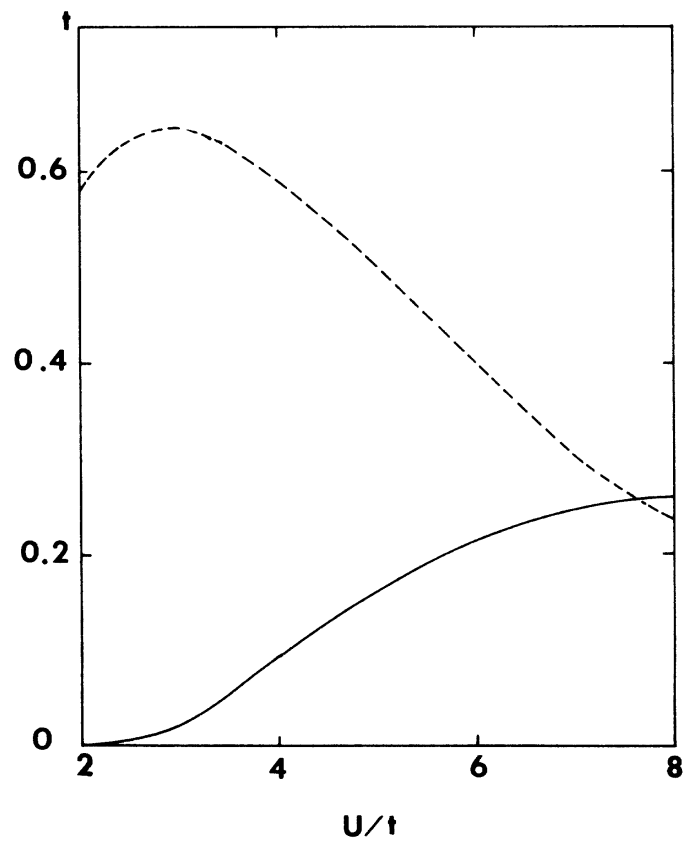

FIG. 2. The attractive potential $V$ [Eq. (2.10)] vs $U / t$ is shown by the solid curve. The dashed curve is the $d$-wave component of the attractive potential obtained in the RPA approach (Ref. 6).
Cooper pair could extend over several times of the lattice constant.

The numerical values of $V$ as a function of $U / t$ are shown in Fig. 2 by the solid curve in the intermediate coupling regime $U \leq 8 t$. One can see that the attractive potential obtained in the present paper is generally reduced as compared with the RPA result ${ }^{6}$ shown by the dashed curve in Fig. 2, which corresponds to the $d$-wave component. For example, at $U=5 t, V=0.17 t$ and the calculated binding energy for $d$-wave pairing is $0.004 t$ which is much reduced comparing to $0.019 t$ obtained in the RPA approach; ${ }^{6}$ at $U=7 t$, the binding energy obtained for $d$-wave pairing is $0.009 t$ in contrast with $0.011 t$ obtained in Ref. 6. But when $U>W$ the attraction potential (2.10) becomes stronger than that obtained in the RPA approach. Actually, the former behaves like $1 / U$ in the large- $U$ limit whereas in the latter case, the potential decreases as quickly as $1 / U^{3}$. These discrepancies will be discussed in Sec. IV. Here we note that in the RPA approach, ${ }^{6-8}$ some important diagrams have been totally neglected and the spin-bag effect has not been included appropriately in the effective interaction. Although the p-wave components in $H_{\mathbf{k}^{\prime}, \mathbf{k}}^{\prime}$ [Eq. (2.9)] have equal weights as the $d$-wave component, the binding energy of $p$-wave pairing is found to be quite small due to its much lesser density of states as compared to the $d$-wave channel.

It is noted that a similar calculation could be carried out for the case of two spin bags with parallel spins. The attractive interaction is found to be very weak, consistent with the result of the RPA approach. ${ }^{6}$

\section{CONCLUSION AND DISCUSSION}

In the itinerant approach, the "parent" antiferromagnetic insulator is a commensurate spin-density-wave state. A hole doped into such a system will induce a local distortion surrounding it in the SDW background. Such distortion is described by the particle-hole excitations. In the weak and intermediate coupling regime $(U<W=8 t)$ it is shown that only the excitation involving one particle-hole pair which couples strongly with the doped hole is predominantly important. A spin-bag wave function is therefore constructed in this regime. The "bag" size is found quite small with its profile extending over from a center site only to its four nearest-neighbor sites. As discussed in Sec. II, more particle-hole excitations will be involved in the spin-bag entity when $U>W$. These excitations result in a spin disordering around the doped hole and make the size of the spin bag become large in the strong-coupling regime.

With obtaining a single spin-bag state, one could construct the free-spin-bag representation and discuss the effective coupling between the spin bags. Such an interaction between two spin bags (with antiparallel spin) is shown to be attractive, which is $p$-wave and $d$-wave like as the consequence of the existence of two sublattices. The potential $V$ [Eq. (2.10)] as a function of $U$ has been shown by the solid curve in Fig. 2 .

A similar conclusion, though with different physics involved, has been also reached in the RPA approach. ${ }^{6-8}$ 


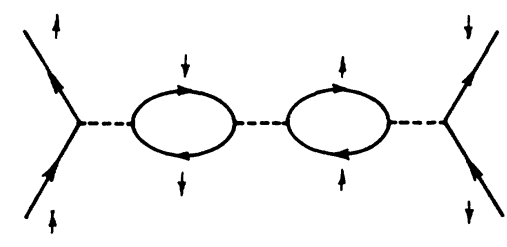

FIG. 3. The lowest order (actually the most important) process of the exchange of the amplitude fluctuation in the RPA.

In the RPA the most important contribution to the attractive interaction comes from the direct exchange of the amplitude fluctuations. Figure 3 shows the lowest order diagram of such a process giving a leading order of $U^{3} \chi_{z z}^{2}(\mathbf{Q})$. The high order contributions are actually negligible in the intermediate- and strong-coupling regime. The $d$-wave component of the attractive potential obtained in the RPA (Ref. 6) is represented by the dashed curve in Fig. 2 which reaches a maximum around $U \sim 3 t$. With the increase of $U$, the potential decreases as $1 / U^{3}$. However, the result of this paper as shown by the solid curve in Fig. 2 is quite different. While the magnitude of the attractive potential is generally reduced in $U<W$ regime, its maximum is pushed to the large- $U$ regime (around $U \sim 10 t$ ) and decreases as $1 / U$ in the large- $U$ limit.

In fact, all of the results in the present paper could be obtained in the equation-of-motion formalism. There one could show that not only the RPA process of Fig. 3 is present in the effective interaction [Eq. (2.9)] but those processes shown in Fig. 4 also contribute to the attractive potential. Their total contribution to $H_{\mathbf{k}^{\prime}, \mathbf{k}}^{\prime}$ is given by

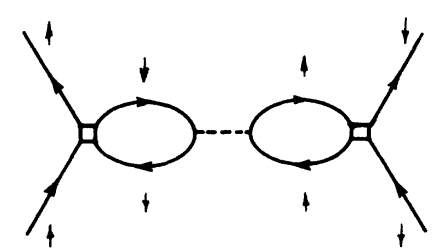

(a)

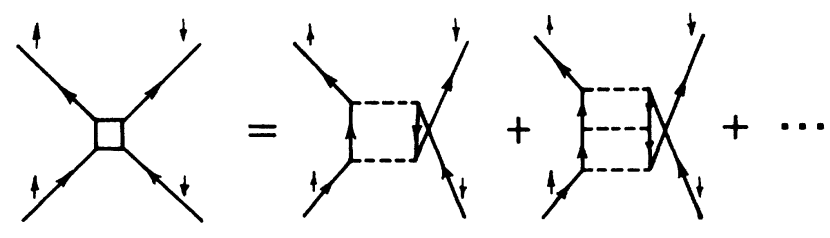

(b)

FIG. 4. A set of processes involved in the attractive potential of Eq. (2.9), which in fact is dominant over the process shown in Fig. 2. the first term in Eq. (2.10) which is much larger than the RPA result. ${ }^{6}$ This means that the contribution coming from Fig. 4 is dominant over the exchange of the simple amplitude fluctuations of Fig. 3 which is emphasized in the RPA calculations of Refs. 4 and 6-8. It is noted that the most important contribution to the spin-bag energy as discussed in Sec. II also comes from similar diagrams shown in Fig. 4(b). These diagrams involve the low energy spin-flip excitations. Therefore, both the strong spinbag effect and the attractive interaction between spin bags are closely related to the low-lying spin-flip excitations. But this does not imply that the itinerant approach is simply in accordance with the strong-coupling approach, as it should be pointed out that the processes of Fig. 4 still involve the exchange of the amplitude and charge fluctuations, with the spin-flip processes appearing in the vertices. On the other hand, under the condition of $\left|\varepsilon_{\mathbf{k}}\right|,\left|\varepsilon_{\mathbf{k}^{\prime}}\right|<\Delta$, the direct exchange of the spin-flip fluctuations still have a negligible contribution to $H_{\mathbf{k}^{\prime}, \mathbf{k}}^{\prime}$, in agreement with the RPA approach. ${ }^{4,6-8}$ A repulsive contribution is also present in $H_{\mathbf{k}^{\prime}, \mathbf{k}}^{\prime}$ or $V$ [the second and third terms on the right side of Eq. (2.10)]. It involves the vertex correction to the direct Coulomb interaction of the doped hole. The lowest order diagram is shown in Fig. 5, which is not present in the simple RPA approach. An intuitive way to understand this interaction is to note that, while the on-site Coulomb interaction is reduced in the SDW background due to the existence of two sublattices, it is restored within the "bag" which suppresses the local antiferromagnetic ordering or sublattices in it. Therefore, there is an extra Coulomb repulsion when two spin bags temporarily share a common bag. For this reason, the total attractive interaction is much reduced in the weak-coupling regime as compared to the RPA result, as Fig. 2 shows. On the other hand, with the increase of $U$, the attractive interaction is enhanced due to the contribution of the low-lying spin-flip processes appearing in the vertices.

Therefore, from the nondiagrammatical approach in this paper, we have learned that the simple calculation based on the RPA carried out in Refs. 6-8 is not enough and the vertex correction and higher order vertices involving spin-flip excitations should be both included to get a reliable result in a diagrammatical approach. Although our calculations are carried out in the half-filled limit where the long-range antiferromagnetic ordering is well defined, the general features of the spin bags and their interaction, such as their small size and the nearest-

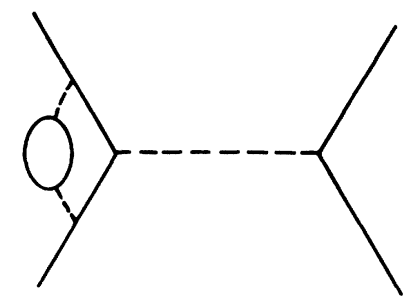

FIG. 5. The Coulomb interaction with the lowest order vertex correction. 
neighbor attractive coupling, enable one to expect that even in the large doping case with disappearing of the long-range order, the spin-bag picture probably still works qualitatively as long as the local antiferromagnetic order exists.

\section{ACKNOWLEDGMENTS}

We would like to acknowledge X. Y. Chen and W. P. $\mathrm{Su}$ for helpful discussions. The present work is supported by the Texas Center for superconductivity at the University of Houston under the Prime Grant No. MDA-97288-G-0002 (Defense Advanced Research Project Agency, TX), and a grant from the Robert A. Welch Foundation (Houston, TX).

\section{APPENDIX A}

The spin-bag operator $\hat{\psi}_{\mathbf{k} \sigma}$ is expressed in the lattice representation by Eq. (1.30), in which the sublattice is chosen such that $\sigma p_{j}=+1$. Explicitly, $\hat{\psi}_{\mathrm{k} \uparrow}$ and $\widehat{\psi}_{\mathrm{k} \downarrow}$ are written in the following form:

$\hat{\psi}_{\mathbf{k} \uparrow}=\sum_{l}^{\prime} \frac{e^{i \mathbf{k} \cdot \overline{\mathbf{R}}_{l}}}{\sqrt{N / 2}}\left(\sin \theta_{k}+\cos \theta_{\mathbf{k}} \beta_{l \downarrow}^{\dagger} \gamma_{l \downarrow}\right) \alpha_{l \uparrow}$,
$\hat{\psi}_{-\mathbf{k} \downarrow}=\sum_{m}^{\prime} \frac{e^{-i \mathbf{k} \cdot \tilde{\mathbf{R}}_{m}}}{\sqrt{N / 2}}\left(\sin \theta_{k}-\cos \theta_{\mathbf{k}} \widetilde{\beta}_{m \downarrow}^{\dagger} \widetilde{\gamma}_{m \downarrow}\right) \widetilde{\alpha}_{m \uparrow}$,

where $\overline{\mathbf{R}}_{l}$ and $\widetilde{\mathbf{R}}_{m}$ refer to the different sublattices, respectively, and one could show according to Eqs. (1.11a) and $(1.11 \mathrm{~b})$ that

$$
\left\{\widetilde{\alpha}_{m \sigma}^{\dagger}, \alpha_{l \sigma}\right\}=\left\{\widetilde{\beta}_{m \sigma}^{\dagger}, \beta_{l \sigma}\right\}=\frac{2}{N} \sum_{\mathbf{k}}^{\prime} e^{-i \mathbf{k} \cdot\left(\tilde{\mathbf{R}}_{m}-\overline{\mathbf{R}}_{l}\right)} .
$$

By the definition of Eq. (2.2) as well as Eqs. (A1) and (A2), one obtains

$$
\left\langle\mathbf{k}^{\prime} \uparrow,-\mathbf{k}^{\prime} \downarrow \mid \mathbf{k} \uparrow,-\mathbf{k} \downarrow\right\rangle=\delta_{\mathbf{k}, \mathbf{k}^{\prime}}-\frac{2}{N}\left[\sin \theta_{k} \cos \theta_{k} \cdot \sin \theta_{\mathbf{k}^{\prime}} \cos \theta_{\mathbf{k}^{\prime}} C_{1}\left(\mathbf{k}, \mathbf{k}^{\prime}\right)+\cos ^{2} \theta_{\mathbf{k}} \cos ^{2} \theta_{\mathbf{k}^{\prime}} C_{2}\left(\mathbf{k}, \mathbf{k}^{\prime}\right)\right],
$$

where

$$
C_{1}\left(\mathbf{k}, \mathbf{k}^{\prime}\right)=2 C(\mathbf{k}) C\left(\mathbf{k}^{\prime}\right)
$$

with

$$
C(\mathbf{k})=\frac{1}{\Gamma_{0}} \sum_{j} e^{i \mathbf{k} \cdot \mathbf{R}_{j}} p_{j} B_{+j}^{3}(0) \frac{u_{\mathbf{k}}-p_{j} v_{\mathbf{k}}}{\sqrt{2}} .
$$

$\boldsymbol{B}_{+j}^{3}(0)$ is a very localized function of $\mathbf{R}_{j}$ and actually its value is non-negligible only at $\mathbf{R}_{j}=0$ and $\mathbf{R}_{j}=a( \pm \mathbf{i}, \pm \mathbf{j})$; here $a$ is the lattice constant. Around the magnetic zone boundary where $\left|\varepsilon_{\mathbf{k}}\right|<\Delta$, one has approximately $u_{\mathrm{k}} \sim v_{\mathrm{k}} \sim 1 / \sqrt{2}$ [Eqs. (1.6a) and (1.6b)] and thus the factor

$$
\left(u_{\mathbf{k}}-p_{j} v_{\mathbf{k}}\right) / \sqrt{2} \sim\left(1-p_{j}\right) / 2
$$

which becomes vanishing at $\mathbf{R}_{j}=0$ as $p_{j}=+1$. Therefore, $C(\mathbf{k})$ reduces to

$$
C(\mathbf{k}) \simeq+B_{+j}^{3}(0)_{|\mathbf{R},|=a} \frac{\varepsilon_{\mathbf{k}}}{\Gamma_{0} t}
$$

when $\left|\varepsilon_{\mathbf{k}}\right|<\Delta$. For example, at $U=2 t, \quad C(\mathbf{k})$ $\simeq 0.023 \varepsilon_{\mathrm{k}} / \Delta$; etc. Hence, under the condition $\left|\varepsilon_{\mathbf{k}}\right|,\left|\varepsilon_{\mathbf{k}^{\prime}}\right|<\Delta, \quad C_{1}\left(\mathbf{k}, \mathbf{k}^{\prime}\right)=2 C(\mathbf{k}) C\left(\mathbf{k}^{\prime}\right)$ could be simply neglected and one finds

$$
\left\langle\mathbf{k}^{\prime} \uparrow,-\mathbf{k}^{\prime} \downarrow \mid \mathbf{k} \uparrow,-\mathbf{k} \downarrow\right\rangle \sim-\frac{2}{N}\left(\cos ^{4} \theta\right) C_{2}\left(\mathbf{k}, \mathbf{k}^{\prime}\right)
$$

for $\mathbf{k} \neq \mathbf{k}^{\prime}$, where $\theta_{\mathbf{k}} \sim \theta_{\mathbf{k}^{\prime}}$ is denoted by $\theta$ and $C_{2}\left(\mathbf{k}, \mathbf{k}^{\prime}\right)$ is given by

$$
C_{2}\left(\mathbf{k}, \mathbf{k}^{\prime}\right)=\frac{2}{N} \sum_{l l^{\prime}}^{\prime} e^{-i\left(\mathbf{k}-\mathbf{k}^{\prime}\right) \cdot\left(\overline{\mathbf{R}}_{l}-\widetilde{\mathbf{R}}_{l^{\prime}}\right)}\left(2-D_{l, l^{\prime}}^{2}\right) D_{l, l^{\prime}}^{2},
$$

in which $D_{l, l^{\prime}}$ is defined in Eq. (2.6).

\section{APPENDIX B}

The interaction matrix element $H_{\mathbf{k}^{\prime}, \mathbf{k}}^{\prime}$ is determined by Eq. (2.4). On the right-hand side of Eq. (2.4), the first two terms involve the operator $\hat{A}_{\mathrm{k} \sigma}$ which is defined through Eq. (2.1). $\hat{A}_{\mathrm{k} \sigma}$ is an incoherent part of the motion of the spin bag which has a negligible effect on the spin bag's self-energy. But with the presence of another spin bag, $\hat{A}_{\mathrm{k} \sigma}$ is important to the correlation between these two spin bags.

As Eq. (2.1) shows, $\hat{A}_{\mathrm{k} \sigma}$ is determined by the commutator $\left[H, \hat{\psi}_{\mathrm{k} \sigma}\right]$ after extracting out the diagonal part $\widetilde{E}_{\mathbf{k}} \hat{\psi}_{\mathbf{k} \sigma}$. $\hat{A}_{\mathbf{k} \sigma}$ could be written in two parts: $\hat{A}_{\mathbf{k} \sigma}=A_{\mathbf{k} \sigma}^{0}+A_{\mathrm{k} \sigma}$, in which $A_{\mathrm{k} q}^{0}$ is deduced from $\left[H_{0}, \hat{\psi}_{\mathrm{k} \sigma}\right]$, while $A_{\mathrm{k} \sigma}^{f}$ is from $\left[H_{f}, \hat{\psi}_{\mathrm{k} \sigma}\right] . A_{\mathrm{k} \sigma}^{0}$ represents an incoherent propagation of the spin bag, with the incoherent hoppings of the particle and holes involved in the spin-bag entity, which actually has no effect on other spin bags (except an exchange process which is negligible, similar to the discussion of the orthogonal condition of state $|\mathbf{k}, \uparrow,-\mathbf{k} \downarrow\rangle$ for different $\mathbf{k}$ 's in Appendix A). Therefore, one finds in $\hat{A}_{\mathbf{k} \sigma}$, only the terms coming from [ $H_{f}, \hat{\psi}_{\mathrm{k} \sigma}$ ] contribute to $H_{\mathbf{k}^{\prime}, \mathbf{k}}^{\prime}$.

$A \hat{\mathrm{k}}_{\sigma}$ is related to the commutator $\left[H_{f}, \hat{\psi}_{\mathrm{k} \sigma}\right]$. In $\hat{\psi}_{\mathrm{k} \sigma}$, there are two terms: One is the single-hole channel, $\sin \theta_{\mathbf{k}} \Sigma_{l}^{\prime}\left(e^{-i \mathbf{k} \cdot \overline{\mathbf{R}}_{l}} / \sqrt{N / 2}\right) \alpha_{l \sigma}$, and the other one is the three-particle-hole channel, $\sigma \cos \theta_{k} \Sigma_{l}^{\prime}\left(e^{-i \mathbf{k} \cdot \overline{\mathbf{R}}_{l}} /\right.$ $\sqrt{N / 2}) \beta_{l-\sigma}^{\dagger} \gamma_{l-\sigma} \alpha_{l \sigma}$, as shown by Eq. (1.30). The contribution of the single-hole part of $\hat{\psi}_{\mathbf{k} \sigma}$ to $\left[H_{f}, \hat{\psi}_{\mathbf{k} \sigma}\right]$ is given as follows: 


$$
\begin{aligned}
& {\left[H_{f}, \sin \theta_{k} \sum_{l}^{\prime} \frac{e^{-i \mathbf{k} \cdot \overline{\mathbf{R}}_{l}}}{\sqrt{N / 2}} \alpha_{l \sigma}\right]=\sin \theta_{k} \sum_{l}^{\prime} \frac{e^{-i \mathbf{k} \cdot \overline{\mathbf{R}}_{l}}}{\sqrt{N / 2}} U} \\
& \times \sum_{j} \sum_{l^{\prime} m n} \cdot\left[B_{\sigma j}(l) B_{\sigma j}\left(l^{\prime}\right) B_{-\sigma j}(m) B_{-\sigma j}(n) \alpha_{l^{\prime} \sigma} \alpha_{m-\sigma} \alpha_{n-\sigma}^{\dagger}\right. \\
& +p_{j} B_{\sigma_{J}}(l) \boldsymbol{B}_{-\sigma j}\left(l^{\prime}\right) \boldsymbol{B}_{-\sigma J}(m) \boldsymbol{B}_{-\sigma j}(n) \beta_{l^{\prime} \sigma} \alpha_{m-\sigma} \alpha_{n-\sigma}^{\dagger} \\
& -B_{\sigma J}(l) B_{\sigma_{J}}\left(l^{\prime}\right) \boldsymbol{B}_{\sigma_{J}}(m) \boldsymbol{B}_{\sigma j}(n) \alpha_{l^{\prime} \sigma} \beta_{n-\sigma}^{+} \beta_{m-\sigma} \\
& -p_{J} B_{\sigma j}(l) B_{-\sigma J}\left(l^{\prime}\right) B_{\sigma j}(m) B_{\sigma j}(n) \beta_{l^{\prime} \sigma} \beta_{n-\sigma}^{\dagger} \beta_{m-\sigma} \\
& -p_{J} B_{\sigma J}(l) B_{\sigma j}\left(l^{\prime}\right) B_{\sigma j}(m) B_{-\sigma J}(n) \alpha_{l^{\prime} \sigma} \alpha_{n-\sigma}^{\dagger} \beta_{m-\sigma} \\
& -p_{j} B_{\sigma j}(l) B_{\sigma j}\left(l^{\prime}\right) \boldsymbol{B}_{\sigma j}(m) \boldsymbol{B}_{-\sigma J}(n) \alpha_{l^{\prime} \sigma} \beta_{m-\sigma}^{\dagger} \alpha_{n-\sigma} \\
& -B_{\sigma j}(l) B_{-\sigma j}\left(l^{\prime}\right) B_{\sigma j}(m) B_{-\sigma j}(n) \beta_{l^{\prime} \sigma} \beta_{m-\sigma}^{\dagger} \alpha_{n-\sigma} \\
& \left.-B_{\sigma j}(l) B_{-\sigma j}\left(l^{\prime}\right) B_{\sigma j}(m) B_{-\sigma j}(n) \beta_{l^{\prime} \sigma} \alpha_{n-\sigma}^{\dagger} \beta_{m-\sigma}\right] .
\end{aligned}
$$

For clarity, Eq. (B1) is shown diagrammatically in Fig. 6(a). There, the incoming hole (initial state) with spin $\sigma$ enters from the left with a particle-hole pair carrying spin $-\sigma$ excited in the final state (right side). Each line represents a particle (creation operator) or hole (annihilation operator), distinguished by a right-directing or leftdirecting arrow. These particle (hole) lines could be in either band, corresponding to the numerous terms in Eq. (B1). The dashed line in Fig. 6(a) is the interaction line.

The expression

$$
\left[H_{f}, \sigma \cos \theta_{k} \Sigma_{l}^{\prime} \frac{e^{-i \mathbf{k} \cdot \overline{\mathbf{R}}_{l}}}{\sqrt{N / 2}} \beta_{l-\sigma}^{\dagger} \gamma_{l-\sigma} \alpha_{l \sigma}\right]
$$

could be similarly calculated. For simplicity, its lengthy expression will not be present here; however, its diagram-

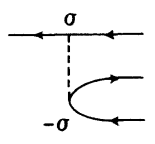

(a)

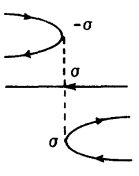

(e)

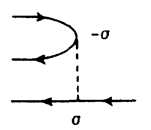

(b)

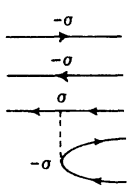

(f)

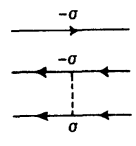

(c)

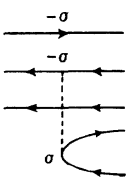

(g)

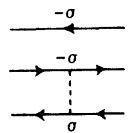

(d)

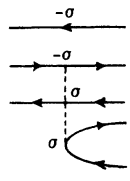

(h)
FIG. 6. The diagrammatical representation of $\left[H_{f}, \hat{\psi}_{\mathbf{k} \sigma}\right]$ which are classified in terms of the different vertices. The leftside lines in each diagram are the incoming particle and holes of $\widehat{\psi}_{\mathbf{k} \sigma}$ and the right-side lines represent the outcoming particles and holes of $\left[H_{f}, \hat{\psi}_{\mathbf{k} \sigma}\right]$. The particle (hole) is denoted by the right- (left-)directing arrow. matical representations are shown in Figs. 6(b)-6(h), just as Eq. (B1) is shown in Fig. 6(a).

Firstly, we consider those terms in $\left[H_{f}, \hat{\psi}_{\mathrm{k} \sigma}\right]$ which give nonvanishing results when acting on $|0\rangle$, and are denoted by $\left[H_{f}, \hat{\psi}_{\mathbf{k} \sigma}\right]_{a}$. It would contribute both to the diagonal part $2 \widetilde{E}_{\mathbf{k}}\left\langle\mathbf{k}^{\prime} \uparrow,-\mathbf{k}^{\prime} \downarrow \mid \mathbf{k} \uparrow,-\mathbf{k} \downarrow\right\rangle$ as well as $H_{\mathbf{k}^{\prime}, \mathbf{k}}^{\prime}$, through the matrix elements $\left\langle\mathbf{k}^{\prime} \uparrow,-\mathbf{k}^{\prime} \downarrow\right|\left[H_{f}\right.$, $\left.\hat{\psi}_{\mathbf{k} \uparrow}\right]_{a} \hat{\psi}_{-\mathbf{k} \downarrow}|0\rangle$ and $\left\langle\mathbf{k}^{\prime} \uparrow,-\mathbf{k}^{\prime} \downarrow\left|\hat{\psi}_{k \uparrow}\left[H_{f}, \hat{\psi}_{-\mathbf{k} \downarrow}\right]_{a}\right| 0\right\rangle$.

For $\left[H_{f}, \hat{\psi}_{\mathbf{k} \sigma}\right]_{a}$, the right-side particle lines in Fig. 6 should be all in the upper band (denoted by $\beta^{+}$) to give a nonzero result when acting on $|0\rangle$, while the hole lines in the right side are all in the lower band (denoted by $\alpha$ ). The processes shown in Figs. 6(a) and 6(b) simply correspond to the transition from the single-hole state $|\mathbf{k} \sigma\rangle$ to the excitation state $\left|\phi_{\mathbf{k} \sigma}\right\rangle$ (see Sec. II) and vice versa. The process of $\left|\phi_{\mathrm{k} \sigma}\right\rangle \rightarrow\left|\phi_{\mathrm{k} \sigma}\right\rangle$ is included in Figs. 6(c) and 6(d). All these terms are essential to the spin-bag solution discussed in Sec. II, or to the diagonal part $2 \widetilde{E}_{\mathbf{k}}\left\langle\mathbf{k}^{\prime} \uparrow,-\mathbf{k} \downarrow \mid \mathbf{k} \uparrow,-\mathbf{k} \downarrow\right\rangle$ here. Now one can see the approximations made in Sec. II to obtain the spin-bag solution (1.29)-(1.33): A truncation is made by neglecting the higher order contributions of Figs. 6(f)-6(h). Figure 6(e) has been also neglected as its very small matrix element as compared to that of Figs. 6(c) and 6(d). On the other

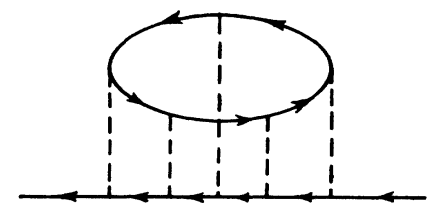

FIG. 7. A typical process involved in the full-time development of one doped hole's motion, which is a combination of the vertices of Figs. 6(a)-6(d). 
hand, the process $\left|\phi_{\mathrm{k} \sigma}\right\rangle \rightarrow\left|\phi_{\mathrm{k} \sigma}^{\prime}\right\rangle$ (which is orthogonal to $\left.\left|\phi_{\mathrm{k} \sigma}\right\rangle\right)$ in Figs. 6(c) and 6(d) is also neglected, as its matrix element is quite small as compared to $\left\langle\phi_{\mathbf{k} \sigma}\left|H_{f}\right| \phi_{\mathbf{k} \sigma}\right\rangle$. It is noted that the elementary processes shown in Fig. 6 may occur in any interval of time during the full time development, and the retained diagrams, Figs. 6(a)-6(d), could compose, for example, such process as shown in
Fig. 7 in the self-energy of the spin bag.

Except for the above-discussed contributions to the diagonal part $2 \widetilde{E}_{k}\left\langle\mathbf{k}^{\prime} \uparrow,-\mathbf{k}^{\prime} \downarrow \mid \mathbf{k} \uparrow,-\mathbf{k} \downarrow\right\rangle,\left[H_{f}, \widehat{\psi}_{\mathbf{k} \sigma}\right]_{a}$ also contribute to the correlations between two spin bags, i.e., $H_{\mathbf{k}^{\prime}, \mathbf{k}}^{\prime}$. For example, the term shown in Fig. 6(e) has a non-negligible contribution to $H_{\mathbf{k}^{\prime}, \mathbf{k}}^{\prime}$ to the order of $\cos ^{2} \theta$ :

$$
\begin{aligned}
& -U \sin \theta_{\mathbf{k}} \cos \theta_{\mathbf{k}} \sin \theta_{\mathbf{k}^{\prime}} \cos \theta_{\mathbf{k}^{\prime}} \frac{4}{N^{2}} \sum_{l l^{\prime}}^{\prime} e^{-l\left(\mathbf{k}-\mathbf{k}^{\prime}\right) \cdot\left(\overline{\mathbf{R}}_{l}-\widetilde{\mathbf{R}}_{l^{\prime}}\right)} \sum_{j} B_{+j}(l) \widetilde{B}_{-j}\left(l^{\prime}\right)\left[2 A_{j}(l) \tilde{A}_{j}\left(l^{\prime}\right)\right. \\
& -D_{l, l^{\prime}}\left(\frac{u_{\mathbf{k}^{\prime}}+p_{j} v_{\mathbf{k}^{\prime}}}{\sqrt{2}} A_{j}(l) \cdot e^{i \mathbf{k}^{\prime} \cdot\left(\mathbf{R}_{j}-\overline{\mathbf{R}}_{l}\right)}\right. \\
& \left.\left.+\frac{u_{\mathbf{k}}{ }^{\prime}-p_{j} v_{\mathbf{k}^{\prime}}}{\sqrt{2}} \widetilde{A}_{j}\left(l^{\prime}\right) e^{-i \mathbf{k}^{\prime} \cdot\left(\mathbf{R},-\tilde{\mathbf{R}}_{l^{\prime}}\right)}\right]\right]
\end{aligned}
$$

in which $\overline{\mathbf{R}}_{l}\left(\widetilde{\mathbf{R}}_{l^{\prime}}\right)$ corresponds to the sublattice with total spin up (down) and the functions $D_{l, l^{\prime}}, A_{j}(l)$, and $\widetilde{A}_{j}\left(l^{\prime}\right)$ are defined by Eqs. (2.6)-(2.8) in Sec. III. Such a contribution is diagrammatically shown in Figs. 8(a) and 8(b), where each brace on either side means that the lines included belong to the same spin bag. We note that in the full time development, Figs. 8(a) and 8(b) could produce the diagrams of Figs. $8(\mathrm{c})$ and $8(\mathrm{~d})$ by combining with Fig. 6(a)-6(d). On the other hand, it is found that the terms corresponding to Figs. 6(c) and 6(d) do not contribute to $H_{\mathbf{k}^{\prime}, \mathbf{k}}^{\prime}$ except an exchange process which is negligible in analogy with the discussion in Appendix A. Figures $6(f)-6(h)$ are also neglected as their contributions to $H_{\mathbf{k}^{\prime}, \mathbf{k}}^{\prime}$ are at least of order of $\cos ^{3} \theta$.

Next, we consider those terms in $\left[H_{f}, \hat{\psi}_{\mathbf{k} \sigma}\right]$ which give the vanishing results when acting directly on $|0\rangle$, which are denoted by $\left[H_{f}, \hat{\psi}_{\mathrm{k} \sigma}\right]_{b}$. Obviously, these terms all belong to $A_{\mathrm{k} \sigma}$ and appear in $H_{\mathbf{k}^{\prime}, \mathrm{k}}^{\prime}$ through the matrix ele- ment $\left\langle\mathbf{k}^{\prime} \uparrow,-\mathbf{k}^{\prime} \downarrow\left|\left[H_{f}, \widehat{\psi}_{\mathbf{k} \uparrow}\right]_{b} \hat{\psi}_{-\mathbf{k} \downarrow}\right| 0\right\rangle$ (evidently, $\left\langle\mathbf{k}^{\prime} \uparrow\right.$, $\left.\left.-\mathbf{k}^{\prime} \downarrow\left|\hat{\psi}_{\mathbf{k} \uparrow}\left[H_{f}, \widehat{\psi}_{\mathbf{k} \downarrow}\right]_{b}\right| 0\right\rangle=0\right)$.

The contributions of $\left[H_{f}, \hat{\psi}_{\mathbf{k} \uparrow}\right]_{b}$ to $H_{\mathbf{k}^{\prime}, \mathbf{k}}^{\prime}$ are diagrammatically shown in Figs. $9(\mathrm{a})-9(\mathrm{k})$. Of them, Figs. 9(a)-9(d) come from those terms in $\left[H_{f}, \hat{\psi}_{\mathbf{k} \uparrow}\right]_{b}$ which are corresponding to Fig. 6(a). Figure $9(\mathrm{e})$ is the contribution of $\left[H_{f}, \hat{\psi}_{\mathbf{k} \uparrow}\right]_{b}$ from Fig. 6(b). Figures $9(\mathrm{f})$ and $9(\mathrm{~g})$ are from Fig. 6(d) and Fig. 9(h) is from Fig. 6(e). And Figs. 9(i)-9(k) correspond to the contributions to $\left[H_{f}, \widehat{\psi}_{\mathbf{k} \uparrow}\right]_{b}$ shown in Figs. 6(f) and 6(h), respectively. All other contributions of $\left[H_{f}, \hat{\psi}_{\mathbf{k} \uparrow}\right]_{b}$ to $H_{\mathbf{k}^{\prime}, \mathbf{k}}^{\prime}$ are either zero of the order higher than $\cos ^{2} \theta$, which are presumably negligible as discussed in Sec. III.

For example, Fig. 9(a) represents the direct Coulomb interaction between two quasiholes. The term $\left[H_{f}, \hat{\psi}_{\mathbf{k} \uparrow}\right]_{b}$ which contributes to Fig. 9(a) is shown in Fig. 6(a) and its expression is obtained from Eq. (B1) as follows:

$$
\sin \theta_{k} \sum_{l}^{\prime} \frac{e^{-i \mathbf{k} \cdot \overline{\mathbf{R}}_{l}}}{\sqrt{N / 2}} U \sum_{j} \sum_{l^{\prime} m n}^{\prime} \boldsymbol{B}_{+j}(l) B_{+j}\left(l^{\prime}\right) B_{-j}(m) B_{-j}(n) \alpha_{l^{\prime} \uparrow} \alpha_{m \downarrow} \alpha_{n \downarrow}^{\dagger} .
$$

There are two terms in $\hat{\psi}_{-\mathbf{k} \downarrow}$ and thus two contributions in $\left\langle\mathbf{k}^{\prime} \uparrow,-\mathbf{k}^{\prime} \downarrow\left|\left[H_{f}, \widehat{\psi}_{\mathbf{k} \uparrow}\right]_{b} \widehat{\psi}_{-\mathbf{k} \downarrow}\right| 0\right\rangle$. For the single-hole channel of $\hat{\psi}_{-k \downarrow}$, it is corresponding to Fig. 9(a) and is given by

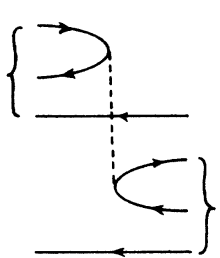

(a)

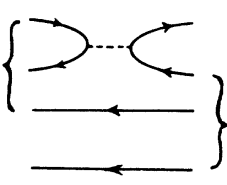

(b)

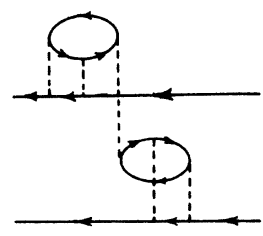

(c)

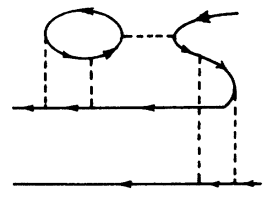

(d)

FIG. 8. (a) and (b): The contributions to $H_{\mathbf{k}^{\prime}, \mathbf{k}}^{\prime}$ from a vertex of $\left[H_{f}, \hat{\psi}_{\mathbf{k} \sigma}\right]_{a}$ shown in Fig. 6(e). (c) and (d): The combinations of (a) and (b) with the vertices of Figs. $6(a)-6(d)$ in the full-time development. 


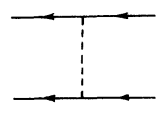

(a)

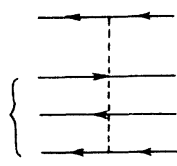

(b)

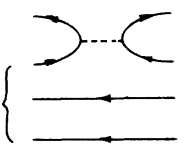

(c)

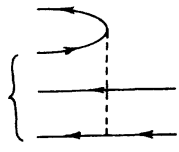

(d)

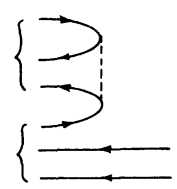

(e)

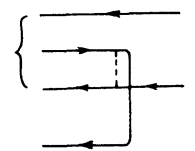

(f)

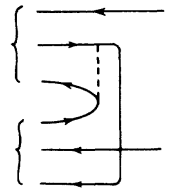

(g)

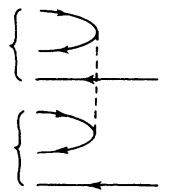

(h)

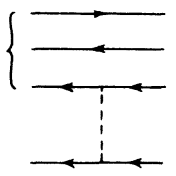

(1)

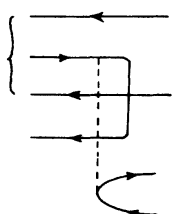

$(1)$

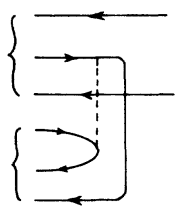

(k)

FIG. 9. The contributions to $H_{\mathbf{k}^{\prime}, \mathbf{k}}^{\prime}$ from the different vertices of $\left[H_{f}, \hat{\psi}_{\mathbf{k} \sigma}\right]_{b}$.

$\sin ^{2} \theta_{k} \sin ^{2} \theta_{k^{\prime}} \frac{4}{N^{2}} \sum_{l l^{\prime} m m^{\prime}}^{\prime} e^{-i \mathbf{k} \cdot\left(\overline{\mathbf{R}}_{l}-\tilde{\mathbf{R}}_{l^{\prime}}\right)+i \mathbf{k}^{\prime} \cdot\left(\overline{\mathbf{R}}_{m}-\tilde{\mathbf{R}}_{m^{\prime}}\right)} \cdot U \sum_{j} B_{+j}(l) B_{+j}(m) \sum_{n}^{\prime} B_{-j}(n)\left[n, m^{\prime}\right] \sum_{n^{\prime}}^{\prime} B_{-j}\left(n^{\prime}\right)\left[n^{\prime}, l\right]$,

where $\left[n, m^{\prime}\right]$ is defined by

$$
\left[n, m^{\prime}\right] \equiv \frac{2}{N} \sum_{k}^{\prime} e^{-i \mathbf{k} \cdot\left(\overline{\mathbf{R}}_{n}-\widetilde{\mathbf{R}}_{m^{\prime}}\right)}
$$

Equation (B4) could be further reduced to the following simple form:

$$
\frac{1}{N} \sin ^{2} \theta_{k} \sin ^{2} \theta_{\mathbf{k}^{\prime}} U\left(u_{\mathbf{k}}^{2}-v_{\mathbf{k}}^{2}\right)\left(u_{\mathbf{k}^{\prime}}^{2}-v_{\mathbf{k}^{\prime}}^{2}\right) .
$$

Thus the contribution of Fig. 9(a) to $H_{\mathbf{k}^{\prime}, \mathbf{k}}^{\prime}$ is vanishingly small near the magnetic zone boundary as $u_{\mathbf{k}}^{2} \simeq v_{\mathbf{k}}^{2}$.

As to the three-particle-hole channel of $\hat{\psi}_{-\mathbf{k} \downarrow}$, (B3) gives the following contribution to $\left\langle\mathbf{k}^{\prime} \uparrow,-\mathbf{k}^{\prime} \downarrow\left|\left[H_{f}, \hat{\psi}_{\mathbf{k} \uparrow}\right]_{b} \hat{\psi}_{-\mathbf{k} \downarrow}\right| 0\right\rangle$ which is corresponding to the diagram of Fig. 9(b):

$$
\begin{aligned}
\sin \theta_{\mathbf{k}} \cos \theta_{\mathbf{k}} \sin \theta_{\mathbf{k}^{\prime}} \cos \theta_{\mathbf{k}^{\prime}} \frac{4}{N^{2}} \sum_{l l^{\prime} m m^{\prime}} e^{-i \mathbf{k} \cdot\left(\overline{\mathbf{R}}_{l}-\tilde{\mathbf{R}}_{l^{\prime}}\right)+i \mathbf{k}^{\prime} \cdot\left(\overline{\mathbf{R}}_{m}-\widetilde{\mathbf{R}}_{m^{\prime}}\right)} U \\
\times \sum_{j} B_{+j}(l) \sum_{l_{1} l_{2} l_{3}}^{\prime} B_{+j}\left(l_{1}\right) B_{-j}\left(l_{2}\right) B_{-j}\left(l_{3}\right) \delta_{m^{\prime}, l^{\prime}}\left[l_{3}, l^{\prime}\right]\left[l_{2}, m^{\prime}\right] \\
\times\left(\delta_{m, l_{1}}-\left\{\widetilde{\gamma}_{m^{\prime} \uparrow}^{\dagger}, \alpha_{l_{1} \uparrow}\right\}\left\{\widetilde{\gamma}_{l^{\prime} \uparrow}, \alpha_{m \uparrow}^{\dagger}\right\}\right) .
\end{aligned}
$$

There are two terms in the last set of parentheses of the above expression. The second term in (B7) comes from an exchange effect which could be shown $\propto C(\mathbf{k}) C\left(\mathbf{k}^{\prime}\right)$ and thus is negligible when $\left|\varepsilon_{\mathbf{k}}\right|,\left|\varepsilon_{\mathbf{k}^{\prime}}\right|<\Delta$ as discussed in Appendix A. For the first term, it could be simplified after using the relation $\sum_{l}^{\prime} B_{-j}(l)\left[l, l^{\prime}\right]=\widetilde{B}_{-j}\left(l^{\prime}\right)$ and the approximation $B_{+j}(l) B_{+j}(m) \simeq B_{+j}^{2}(l) \delta_{l, m}$ in the summation over $j$, that is

$$
\sin \theta_{\mathbf{k}} \cos \theta_{\mathbf{k}} \sin \theta_{\mathbf{k}^{\prime}} \cos \theta_{\mathbf{k}^{\prime}} \frac{4}{N^{2}} \sum_{l l^{\prime}}^{\prime} e^{-i\left(\mathbf{k}-\mathbf{k}^{\prime}\right) \cdot\left(\overline{\mathbf{R}}_{l}-\tilde{\mathbf{R}}_{l^{\prime}}\right)} U \sum_{j} B_{+j}^{2}(l) \widetilde{B}_{-j}^{2}\left(l^{\prime}\right)
$$

All other contributions of $\left[H_{f}, \widehat{\psi}_{\mathbf{k} \uparrow}\right]_{b}$ to $H_{\mathbf{k}^{\prime}, \mathbf{k}}^{\prime}$ could be calculated in this way. We note that the contributions of Fig. 9(d), Fig. 9(e), Fig. 9(f), Fig. 9(g), and Fig. 9(k) are all negligible along the magnetic zone boundary as $\left|\varepsilon_{\mathbf{k}}\right|,\left|\varepsilon_{\mathbf{k}^{\prime}}\right|<\Delta$. And the last result is 


$$
\begin{aligned}
& \left\langle\mathbf{k}^{\prime} \uparrow,-\mathbf{k}^{\prime} \downarrow\left|\left[H_{f}, \hat{\psi}_{\mathbf{k} \uparrow}\right]_{b} \hat{\psi}_{-\mathbf{k} \downarrow}\right| 0\right\rangle=\frac{4}{N^{2}} \sum_{l l^{\prime}}^{\prime} e^{-i\left(\mathbf{k}-\mathbf{k}^{\prime}\right) \cdot\left(\overline{\mathbf{R}}_{l}-\tilde{\mathbf{R}}_{l^{\prime}}\right)} \\
& \times\left\{\sin \theta_{\mathbf{k}} \cos \theta_{\mathbf{k}} \sin \theta_{\mathbf{k}^{\prime}} \cdot \cos \theta_{\mathbf{k}^{\prime}} U\right. \\
& \times \sum_{j} \boldsymbol{B}_{+j}(l) \widetilde{\boldsymbol{B}}_{-j}\left(l^{\prime}\right) i \\
& \times\left[2 B_{+j}(l) \widetilde{B}_{-j}\left(l^{\prime}\right)\right. \\
& +D_{l, l^{\prime}}\left(\frac{u_{\mathbf{k}}+p_{j} v_{\mathbf{k}}}{\sqrt{2}} A_{j}(l) e^{-i \mathbf{k} \cdot\left(\mathbf{R}_{j}-\tilde{\mathbf{R}}_{l}\right)}\right. \\
& \left.\left.+\frac{u_{\mathbf{k}}-p_{j} v_{\mathbf{k}}}{\sqrt{2}} \widetilde{A}_{j}\left(l^{\prime}\right) e^{i \mathbf{k} \cdot\left(\mathbf{R},-\tilde{\mathbf{R}}_{l^{\prime}}\right)}\right]\right] \\
& -\sin ^{2} \theta_{\mathbf{k}} \cos ^{2} \theta_{\mathbf{k}} \cdot U \\
& \left.\times \sum_{j} B_{+j}(l) \widetilde{B}_{-j}\left(l^{\prime}\right) A_{j}(l) \tilde{A}_{j}\left(l^{\prime}\right)\right\} \text {. }
\end{aligned}
$$

Therefore, the first two terms in $H_{\mathbf{k}^{\prime}, \mathbf{k}}^{\prime}$ [Eq. (2.4)] involving $\hat{A}_{\mathbf{k} \sigma}$ are obtained in Eqs. (B2) and (B9). The last matrix element in Eq. (2.4), i.e., $\left\langle\mathbf{k}^{\prime} \uparrow,-\mathbf{k}^{\prime} \downarrow\left|\hat{\psi}_{\mathbf{k} \uparrow} \widehat{\psi}_{-\mathbf{k} \downarrow} H_{f}\right| 0\right\rangle$, could be regarded as the effective coupling of spin bags through absorbing the virtual background fluctuations. Such a matrix element could be discussed similarly. Under the condition of $\left|\varepsilon_{\mathbf{k}}\right|,\left|\varepsilon_{\mathbf{k}^{\prime}}\right|<\Delta$, and to the order of $\cos ^{2} \theta$, it is obtained

$$
\left\langle\mathbf{k}^{\prime} \uparrow,-\mathbf{k}^{\prime} \downarrow\left|\hat{\psi}_{\mathbf{k} \uparrow} \psi_{-\mathbf{k} \downarrow} H_{f}\right| 0\right\rangle=-\sin ^{2} \theta_{\mathbf{k}} \cos ^{2} \theta_{\mathbf{k}^{\prime}} \frac{4}{N^{2}} \sum_{l, l^{\prime}}^{\prime} e^{-i\left(\mathbf{k}-\mathbf{k}^{\prime}\right) \cdot\left(\overline{\mathbf{R}}_{l}-\widetilde{\mathbf{R}}_{l^{\prime}}\right)} U \sum_{j} B_{+j}(l) \widetilde{B}_{-j}\left(l^{\prime}\right) A_{j}(l) \widetilde{A}_{j}\left(l^{\prime}\right) .
$$

${ }^{1}$ G. Shirane et al., Phys. Rev. Lett. 59, 1613 (1987); J. Tranquada et al., ibid. 60, 156 (1988); M. Sato et al., ibid. 61, 1317 (1988).

${ }^{2}$ K. B. Lyons et al., Phys. Rev. Lett. 60, 732 (1988); R. J. Birgeneau et al., Phys. Rev. B 38, 6614 (1988); R. J. Birgeneau et al., ibid. 39, 2868 (1989).

${ }^{3}$ S. A. Trugman, Phys. Rev. B 37, 1597 (1988); S. Schmitt-Rink, C. M. Varma, and A. G. Ruckenstein, Phys. Rev. Lett. 60, 2793 (1988); B. I. Shraiman, and E. D. Siggia, ibid. 61, 467 (1988); C. L. Kane, P. A. Lee, and N. Read, Phys. Rev. B 39, 6880 (1989); Z. B. Su, Y. M. Li, W. Y. Lai, and L. Yu, Phys. Rev. Lett. 63, 1318 (1989).

${ }^{4}$ J. R. Schrieffer, X.-G. Wen, and S.-C. Zhang, Phys. Rev. Lett.
60, 944 (1988).

${ }^{5}$ W. P. Su, Phys. Rev. B 37, 9904 (1988); H. Y. Choi and E. J. Mele, ibid. 38, 4540 (1988); W. P. Su and X. Y. Chen, ibid. 38, 8879 (1988).

${ }^{6}$ Z. Y. Weng, T. K. Lee, and C. S. Ting, Phys. Rev. B 38, 6561 (1988).

${ }^{7}$ G. Vignale and K. S. Singwi, Phys. Rev. B 39, 2956 (1989).

${ }^{8}$ J. R. Schrieffer, X. G. Wen, and S. C. Zhang, Phys. Rev. B 39, 11663 (1989).

${ }^{9}$ P. W. Anderson, Phys. Rev. 86, 694 (1952).

${ }^{10}$ See, e.g., P. Roman, Advanced Quantum Theory (AddisonWesley, Reading, Mass., 1965).

${ }^{11}$ W. F. Brinkman and T. M. Rice, Phys. Rev. B 2, 1324 (1970). 